\title{
Modular Schur numbers
}

\author{
Jonathan Chappelon* \\ Institut de Mathématiques et de Modélisation de Montpellier \\ Université Montpellier 2, France \\ jonathan.chappelon@um2.fr \\ María Pastora Revuelta Marchena María Isabel Sanz Domínguez \\ Departamento de Matemática Aplicada I \\ Escuela Técnica Superior de Ingeniería de Edificación \\ Universidad de Sevilla, Spain \\ \{pastora, isanz\}@us.es
}

Submitted: May 21, 2012; Accepted: Jun 18, 2013; Published: Jun 30, 2013

Mathematics Subject Classifications: 05C55, 05D10, 11A07, 05A17, 11P81, 11P83

\begin{abstract}
For any positive integers $l$ and $m$, a set of integers is said to be (weakly) $l$-sumfree modulo $m$ if it contains no (pairwise distinct) elements $x_{1}, x_{2}, \ldots, x_{l}, y$ satisfying the congruence $x_{1}+\ldots+x_{l} \equiv y \bmod m$. It is proved that, for any positive integers $k$ and $l$, there exists a largest integer $n$ for which the set of the first $n$ positive integers $\{1,2, \ldots, n\}$ admits a partition into $k$ (weakly) $l$-sum-free sets modulo $m$. This number is called the generalized (weak) Schur number modulo $m$, associated with $k$ and $l$. In this paper, for all positive integers $k$ and $l$, the exact value of these modular Schur numbers are determined for $m=1,2$ and 3 .
\end{abstract}

Keywords: modular Schur numbers; Schur numbers; weak Schur numbers; sumfree sets; weakly sum-free sets

\section{Introduction}

In [8], Guy proposed two unsolved problems in elementary number theory. The first one is the Schur's problem of partitioning integers into sum-free classes (Problem E12). Schur proved in [13] that if the set of the first $k$ ! e positive integers is partitioned into $k$ parts any way, then $x+y=z$ can be solved in integers within one part. Let $\mathrm{S}(k)$ be the largest

${ }^{*}$ Part of this work was carried out during a short postdoctoral research visit at the Instituto de Matemáticas de la Universidad de Sevilla Antonio de Castro Brzezicki (IMUS). 
integer $n$ such that there exists a partition of the first $n$ positive integers $\{1,2, \ldots, n\}$ into $k$ parts with no solution to the equation

$$
x+y=z,
$$

in any part. The exact value of $\mathrm{S}(k)$ is known only for $k \in\{1,2,3,4\}$ and the Problem E12 is to determine it for $k \geqslant 5$. The second unsolved problem proposed by Guy is a modular version of this Schur's problem (Problem E13). It was posed by Abbott and Wang in [2]. Let $\mathrm{T}(k)$ be the largest integer $n$ such that there exists a partition of $\{1,2, \ldots, n\}$ into $k$ parts, with no solution to the congruence

$$
x+y \equiv z \quad(\bmod n+1)
$$

in any part. Abbott and Wang determined that $\mathrm{T}(k)=\mathrm{S}(k)$ for $k \in\{1,2,3,4\}$, and they conjectured that the equality is true for all positive integers $k$. The main purpose of this paper is to study and explicitly determine modular generalizations of Schur numbers.

\subsection{Modular generalized Schur numbers}

Let $k$ be a positive integer and let $S$ be a set of integers. A $k$-partition of $S$ is a set $P=\left\{S_{1}, \ldots, S_{k}\right\}$ of subsets of $S$ such that any element of $S$ is contained in exactly one element of $P$. Let $l$ be a positive integer. A set of integers is said to be $l$-sum-free if it contains no elements $x_{1}, \ldots, x_{l}, y$ satisfying

$$
x_{1}+\ldots+x_{l}=y \text {. }
$$

For every positive integer $k$, the generalized Schur number $\mathrm{S}(k, l)$ is the largest integer $n$ for which the set of the first $n$ positive integers $\{1,2, \ldots, n\}$ admits a $k$-partition into $l$-sum-free sets.

For $l=2$, the numbers $\mathrm{S}(k, 2)=\mathrm{S}(k)$ are known as Schur numbers. They have been introduced by Schur himself in 1916 [13], in order to study a modular version of the Fermat's Last Theorem. He proved that those numbers are always finite, for every positive integer $k$. The first few Schur numbers are given in Table 1.

\begin{tabular}{|c|c|c|c|c|c|c|c|}
\hline$k$ & 1 & 2 & 3 & 4 & 5 & 6 & 7 \\
\hline $\mathrm{S}(k, 2)$ & 1 & 4 & 13 & 44 & $160 \leqslant \cdots \leqslant 305$ & $\geqslant 536$ & $\geqslant 1680$ \\
\hline
\end{tabular}

Table 1: The first few Schur numbers $\mathrm{S}(k, 2)$.

The exact value of $\mathrm{S}(4,2)$ was obtained by Baumert [1]. The lower and upper bounds of $\mathrm{S}(5,2)$ are due to Exoo [6] and Sanz [12], respectively. Finally, the lower bounds of $\mathrm{S}(6,2)$ and $\mathrm{S}(7,2)$ were obtained by Fredricksen and Sweet [7] by considering symmetric sum-free partitions. 
Many generalizations of Schur numbers have appeared since their introduction. In this paper, the generalized Schur numbers that we consider are similarly defined in $[3,10]$. These numbers are always finite (see $[11,10]$ for instance).

Let $m$ be a positive integer. A set of integers is said to be $l$-sum-free modulo $m$ if it contains no elements $x_{1}, \ldots, x_{l}, y$ satisfying

$$
x_{1}+\ldots+x_{l} \equiv y \quad(\bmod m) .
$$

For every positive integer $k$, the generalized Schur number modulo $m$, denoted by $\mathrm{S}_{m}(k, l)$, is the largest integer $n$ for which the set of the first $n$ positive integers $\{1,2, \ldots, n\}$ admits a $k$-partition into $l$-sum-free sets modulo $m$.

Obviously, for every modulus $m$, the inequality

$$
\mathrm{S}_{m}(k, l) \leqslant \mathrm{S}(k, l)
$$

holds because a $l$-sum-free set modulo $m$ of integers is also $l$-sum-free. Moreover, since $m+\ldots+m \equiv m \bmod m$, a $l$-sum-free set of integers modulo $m$ does not contain the integer $m$. Therefore, we have

$$
\mathrm{S}_{m}(k, l) \leqslant m-1 .
$$

For $l=2$, Abbott and Wang investigated in [2] the numbers

$$
\mathrm{T}(k)=\max \left\{n \in \mathbb{N} \mid \mathrm{S}_{n+1}(k, 2)=n\right\},
$$

where $k$ is a positive integer. They obtained that $\mathrm{T}(k)=\mathrm{S}(k, 2)$ for $k \in\{1,2,3,4\}$ and they conjectured that the equality is true for all positive integers $k$.

In this paper, we explicitly determine the modular generalized Schur numbers $\mathrm{S}_{m}(k, l)$ for small values of $m$ : for all moduli $m \in\{1,2,3\}$. For $m=1$, the result is clear. Indeed,

$$
\mathrm{S}_{1}(k, l)=0,
$$

for all $k \geqslant 1$ and $l \geqslant 1$, since every positive integer $x$ verifies $x+\ldots+x \equiv x \bmod 1$ and thus, there does not exist non-empty $l$-sum-free set modulo 1 . For $m=2$ and $m=3$, the exact values of $\mathrm{S}_{m}(k, l)$ are given by the following theorems.

Theorem 1.1. Let $k$ and $l$ be two positive integers. Then,

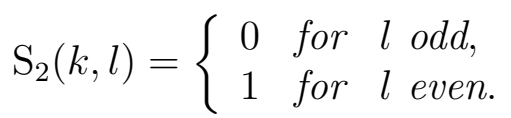

Theorem 1.2. Let $k$ and $l$ be two positive integers. Then,

$$
\mathrm{S}_{3}(k, l)=\left\{\begin{array}{l}
0 \text { for } k \geqslant 1 \text { and } l \equiv 1 \bmod 3, \\
1 \text { for } k=1 \text { and } l \equiv 0,2 \bmod 3, \\
2 \text { for } k \geqslant 2 \text { and } l \equiv 0,2 \bmod 3 .
\end{array}\right.
$$

A simple proof of these theorems will be provided in Section 2 . 


\subsection{Modular generalized weak Schur numbers}

A set of integers is said to be weakly l-sum-free if it contains no pairwise distinct elements $x_{1}, \ldots, x_{l}, y$ satisfying

$$
x_{1}+\ldots+x_{l}=y \text {. }
$$

For every positive integer $k$, the generalized weak Schur number $\operatorname{WS}(k, l)$ is the largest integer $n$ for which the set of the first $n$ positive integers $\{1,2, \ldots, n\}$ admits a $k$-partition into weakly $l$-sum-free sets.

For $l=2$, the numbers $\mathrm{WS}(k, 2)$ are called weak Schur numbers. The first few weak Schur numbers are given in Table 2 .

\begin{tabular}{|c|c|c|c|c|c|c|}
\hline$k$ & 1 & 2 & 3 & 4 & 5 & 6 \\
\hline $\mathrm{WS}(k, 2)$ & 2 & 8 & 23 & 66 & $\geqslant 196$ & $\geqslant 575$ \\
\hline
\end{tabular}

Table 2: The first few weak Schur numbers $\mathrm{WS}(k, 2)$.

The exact value of WS $(4,2)$ was obtained by Blanchard, Harary and Reis [4]. The lower bounds of WS $(5,2)$ and WS $(6,2)$ are due to Eliahou, Marín, Revuelta and Sanz [5].

More generally, the generalized weak Schur numbers are always finite (see [14, 9, 10] for instance). Moreover, the generalized weak Schur numbers appear as a good upper bound for the generalized Schur numbers, since a weakly $l$-sum-free set of integers is also $l$-sum-free. Therefore, we have

$$
\mathrm{S}(k, l) \leqslant \mathrm{WS}(k, l),
$$

for all positive integers $k$ and $l$. A trivial lower bound for the weak Schur numbers is

$$
k l \leqslant \mathrm{WS}(k, l)
$$

because each of the $k$ weakly sum-free sets can contain $l$ distinct integers without solution of the equation $x_{1}+\ldots+x_{l}=y$. Better lower bounds for $\mathrm{WS}(k, l)$ can be found in [12]. A set of integers is said to be weakly l-sum-free modulo $m$ if it contains no pairwise distinct elements $x_{1}, \ldots, x_{l}, y$ satisfying

$$
x_{1}+\ldots+x_{l} \equiv y \quad(\bmod m) .
$$

For every positive integer $k$, the generalized weak Schur number modulo $m$, denoted by $\mathrm{WS}_{m}(k, l)$, is the largest integer $n$ for which the set of the first $n$ positive integers $\{1,2, \ldots, n\}$ admits a $k$-partition into weakly $l$-sum-free sets modulo $m$.

For every modulus $m$, the inequality

$$
\mathrm{WS}_{m}(k, l) \leqslant \mathrm{WS}(k, l)
$$

holds because a weakly $l$-sum-free set modulo $m$ of integers is also weakly $l$-sum-free. Abbott and Wang conjectured that $\mathrm{T}(k)$ is equal to $\mathrm{S}(k, 2)$, for all positive integer $k$. Here, in the weak case, it appears that considering similar numbers than $\mathrm{T}(k)$ is without 
great interest. Indeed, as we can see in Table 3 for $m \in\{1, \ldots, 15\}$, the values of modular generalized weak Schur numbers, for $k=2$ and $l=2$, seem to be very difficult to predict. For $m \geqslant 16$, we have $\mathrm{WS}_{m}(2,2)=\mathrm{WS}(2,2)$ because $\mathrm{WS}_{m}(2,2) \leqslant \mathrm{WS}(2,2)=8$ and because, for two distinct integers $x, y \in\{1, \ldots, 8\}$, we always have $x+y \leqslant 15<m$.

\begin{tabular}{|c|c|c|c|c|c|c|c|c|c|c|c|c|c|c|c|}
\hline$m$ & 1 & 2 & 3 & 4 & 5 & 6 & 7 & 8 & 9 & 10 & 11 & 12 & 13 & 14 & 15 \\
\hline $\mathrm{WS}_{m}(2,2)$ & 4 & 5 & 4 & 5 & 6 & 6 & 7 & 6 & 7 & 7 & 7 & 8 & 8 & 8 & 8 \\
\hline
\end{tabular}

Table 3: The modular generalized weak Schur numbers $\mathrm{WS}_{m}(2,2)$

In this paper, we explicitly determine the modular generalized weak Schur numbers $\mathrm{WS}_{m}(k, l)$ for small values of $m$ : for all moduli $m \in\{1,2,3\}$. For $m=1$, we obtain that

$$
\mathrm{WS}_{1}(k, l)=k l,
$$

for all $k \geqslant 1$ and $l \geqslant 1$, since a weakly $l$-sum-free set modulo 1 has cardinality of at most $l$ because every $l+1$ distinct positive integers $x_{1}, \ldots, x_{l}, y$ verify $x_{1}+\ldots+x_{l} \equiv y \bmod 1$. For $m=2$ and $m=3$ the following theorems will be proved in Section 4 and Section 5 respectively.

Theorem 1.3. Let $k$ and $l$ be two positive integers. Then,

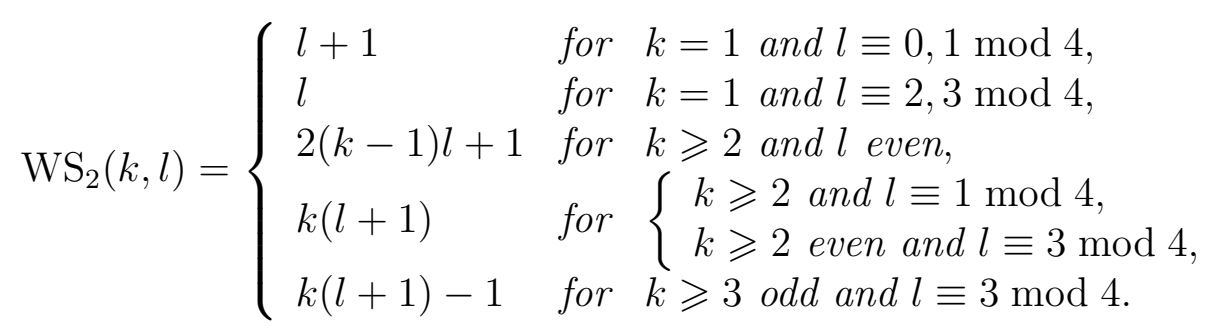

Theorem 1.4. Let $k$ and $l$ be two positive integers. Then,

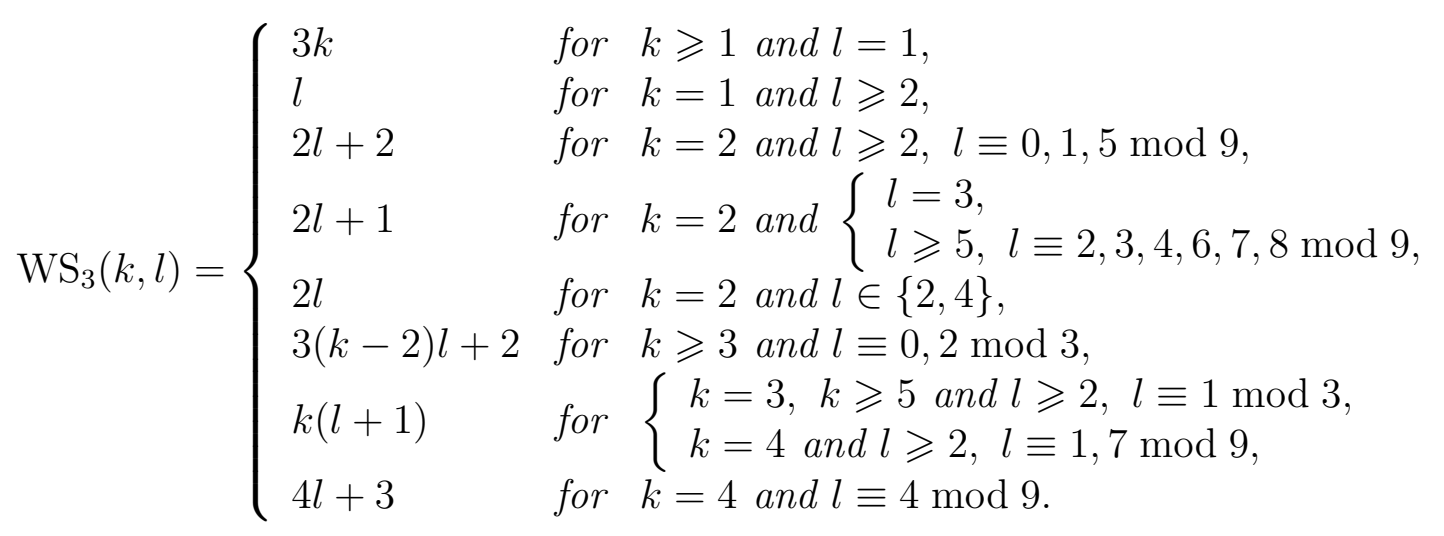

\subsection{Contents}

This paper is organized as follows. A simple proof of Theorems 1.1 and 1.2 is given in Section 2, which completely determines the exact value of the generalized Schur numbers 
modulo 2 and 3. In Section 3, several basic results on the projection of partitions of integers in $\mathbb{Z} / m \mathbb{Z}$, which will be useful in our proofs in the sequel, are introduced. The generalized weak Schur numbers modulo 2 are obtained by proving Theorem 1.3 in Section 4 and the case $m=3$ is settled in Section 5 by proving Theorem 1.4. Remark that Theorems 1.3 and 1.4 are the most difficult results to prove in this paper, much more difficult than Theorems 1.1 and 1.2 whose proofs, in Section 2, are direct. Finally, in Section 6, we discuss open problems.

\section{$2 \quad S_{m}(k, l)$ for the moduli $m=2$ and $m=3$}

We begin this section by proving Theorem 1.1, that is,

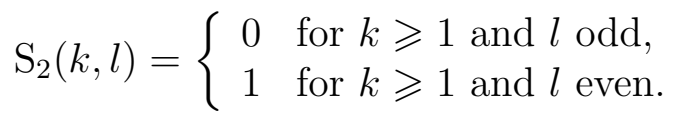

Proof of Theorem 1.1. Let $k$ and $l$ be two positive integers. As already remarked in Section 1 , the inequality $\mathrm{S}_{2}(k, l) \leqslant 1$ holds. Moreover, since $\sum_{i=1}^{l} 1=l$, it follows that the integer 1 belongs to a sum-free set of integers modulo 2 if and only if $l$ is even.

We end with the proof of Theorem 1.2, that is,

$$
\mathrm{S}_{3}(k, l)=\left\{\begin{array}{l}
0 \text { for } k \geqslant 1 \text { and } l \equiv 1 \bmod 3 \\
1 \text { for } k=1 \text { and } l \equiv 0,2 \bmod 3 \\
2 \text { for } k \geqslant 2 \text { and } l \equiv 0,2 \bmod 3
\end{array}\right.
$$

Proof of Theorem 1.2. Let $k$ and $l$ be two positive integers. Since $\sum_{i=1}^{l} 1=l$, it follows that the integer 1 belongs to a $l$-sum-free set of integers modulo 3 if and only if $l \equiv 0$ or $2 \bmod 3$. Thus, we have $\mathrm{S}_{3}(k, l)=0$ when $l \equiv 1 \bmod 3$. Since $\sum_{i=1}^{l} 1=l \equiv 2 \bmod 3$ for $l \equiv 2 \bmod 3$ and $\sum_{i=1}^{l-1} 1+2=l+1 \equiv 1 \bmod 3$ for $l \equiv 0 \bmod 3$, it follows that the integers 1 and 2 cannot belong together to a $l$-sum-free set of integers modulo 3 when $l \equiv 0$ or $2 \bmod 3$. Therefore $\mathrm{S}_{3}(1, l)=1$ for $k=1$ when $l \equiv 0$ or $2 \bmod 3$. Finally, if $k \geqslant 2$ and $l \equiv 0$ or $2 \bmod 3$, then $\mathrm{S}_{3}(k, l) \leqslant 2$ as remarked in Section 1 and $\mathrm{S}_{3}(k, l) \geqslant 2$ because the sets $\{1\}$ and $\{2\}$ are both $l$-sum-free modulo 3 . This leads to the formula $\mathrm{S}_{3}(k, l)=2$ in this case.

\section{$3 \quad$ Projective partitions in $\mathbb{Z} / m \mathbb{Z}$}

Throughout this paper, projections of partitions into $\mathbb{Z} / m \mathbb{Z}$ will be considered. Let

$$
\pi_{m}: \mathbb{Z} \longrightarrow \mathbb{Z} / m \mathbb{Z}
$$

denote the canonical projection map. Let $S$ be a set of integers and let $P=\left\{S_{1}, \ldots, S_{k}\right\}$ be a $k$-partition of $S$. Denote by $\pi_{m}(P)$ the projection of the partition $P$ of $S$, that is the partition $\pi_{m}(P)=\left\{\pi_{m}\left(S_{1}\right), \ldots, \pi_{m}\left(S_{k}\right)\right\}$ of the multiset $\pi_{m}(S)$ of $\mathbb{Z} / m \mathbb{Z}$. 
For example, if we consider the partition

$$
P=\{\{1,2,4,8\},\{3,5,6,7\}\}
$$

of the set $S=\{1, \ldots, 8\}$, then we obtain that

$$
\pi_{2}(P)=\{\{1,0,0,0\},\{1,1,0,1\}\}
$$

is a partition of the multiset $\pi_{2}(S)=\{0,0,0,0,1,1,1,1\}$ of $\mathbb{Z} / 2 \mathbb{Z}$.

As for sets of integers, a multiset of $\mathbb{Z} / m \mathbb{Z}$ is said to be (weakly) $l$-sum-free if it contains no (pairwise distinct) elements $x_{1}, \ldots, x_{l}, y$ satisfying $x_{1}+\ldots+x_{m}=y$ in $\mathbb{Z} / m \mathbb{Z}$.

Proposition 3.1. A set $S$ of integers is (weakly) l-sum-free modulo $m$ if and only if its projection $\pi_{m}(S)$ is a (weakly) l-sum-free multiset of $\mathbb{Z} / m \mathbb{Z}$.

Remark that the partition $P$, of the previous example, is a partition of $S$ into weakly 2 -sum-free sets but it is not a partition of $S$ into weakly 2 -sum-free sets modulo 2 since $\pi_{2}(P)$ is not a partition of $\pi_{2}(S)$ into weakly 2 -sum-free multisets in $\mathbb{Z} / 2 \mathbb{Z}$. Indeed, in each element of $\pi_{2}(P)$, we have three distinct elements satisfying either $0+0=0$ or $1+1=0$ in $\mathbb{Z} / 2 \mathbb{Z}$.

For every multiset $M$ of $\mathbb{Z} / m \mathbb{Z}$, denote by

$$
\mathfrak{m}_{M}: \mathbb{Z} / m \mathbb{Z} \longrightarrow \mathbb{N}
$$

the multiplicity function associated with $M$, that is the function which assigns to each element $x \in \mathbb{Z} / m \mathbb{Z}$ its multiplicity in $M$. Let $|M|$ denote the cardinality of the multiset $M$, that is the number of elements constituting $M$, counted with multiplicity, that is

$$
|M|=\sum_{x \in \mathbb{Z} / m \mathbb{Z}} \mathfrak{m}_{M}(x) \in \mathbb{N}
$$

Obviously, by $\pi_{n}$, each $k$-partition of a set of $n$ positive integers can be associated uniquely with a $k$-partition of a multiset of $n$ terms in $\mathbb{Z} / m \mathbb{Z}$, counted with multiplicity. Conversely, this process is not bijective in general. Indeed, distinct partitions of the same set of integers can be projected on the same partition in $\mathbb{Z} / m \mathbb{Z}$.

Proposition 3.2. Let $m$ and $n$ be two positive integers and let $S=\{1,2, \ldots, n\}$. The exact number of $k$-partitions $P=\left\{S_{1}, \ldots, S_{k}\right\}$ of $S$ that have the same projective $k$ partition $\pi_{m}(P)=\left\{\pi_{m}\left(S_{1}\right), \ldots, \pi_{m}\left(S_{k}\right)\right\}$ is equal to

$$
\prod_{v=0}^{m-1} \prod_{u=1}^{k}\left(\begin{array}{c}
\sum_{w=u}^{k} \mathfrak{m}_{\pi_{m}\left(S_{w}\right)}(v) \\
\mathfrak{m}_{\pi_{m}\left(S_{u}\right)}(v)
\end{array}\right)=\prod_{v=0}^{m-1}\left(\begin{array}{c}
\sum_{w=1}^{k} \mathfrak{m}_{\pi_{m}\left(S_{w}\right)}(v) \\
\mathfrak{m}_{\pi_{m}\left(S_{1}\right)}(v), \mathfrak{m}_{\pi_{m}\left(S_{2}\right)}(v), \ldots, \mathfrak{m}_{\pi_{m}\left(S_{k}\right)}(v)
\end{array}\right)
$$

where $\left(\begin{array}{c}a \\ b_{1}, b_{2}, \ldots, b_{k}\end{array}\right)$ is the multinomial coefficient $\frac{a !}{b_{1} ! b_{2} ! \cdots b_{k} !}$. 
Proof. Consider the euclidean division of $n$ by $m$, that is $n=q m+r$. Let $\varepsilon$ be the function defined by $\varepsilon: \mathbb{Z} / m \mathbb{Z} \longrightarrow\{0,1\}$ where $\varepsilon(v)=1$ for $v \in\{1, \ldots, r\}$ and $\varepsilon(v)=0$ for $v \in\{0, r+1, \ldots, m-1\}$. Thus there is exactly $q+\varepsilon(v)$ integers in $\{1, \ldots, n\}$ whose residue class modulo $m$ is $v \in \mathbb{Z} / m \mathbb{Z}$. We proceed by induction on $u$. Suppose that we have already chosen the integers in the first $u-1$ sets of the $k$-partition $P$. For the set $S_{u}$, we have to choose, for every $v \in \mathbb{Z} / m \mathbb{Z}, \mathfrak{m}_{\pi_{m}\left(S_{u}\right)}(v)$ integers among the remaining $q+\varepsilon(v)-\sum_{w=1}^{u-1} \mathfrak{m}_{\pi_{m}\left(S_{w}\right)}(v)$ integers whose residue class modulo $m$ is $v$. This corresponds to the binomial coefficient

$$
\left(\begin{array}{c}
q+\varepsilon(v)-\sum_{w=1}^{u-1} \mathfrak{m}_{\pi_{m}\left(S_{w}\right)}(v) \\
\mathfrak{m}_{\pi_{m}\left(S_{u}\right)}(v)
\end{array}\right)=\left(\begin{array}{c}
\sum_{w=u}^{k} \mathfrak{m}_{\pi_{m}\left(S_{w}\right)}(v) \\
\mathfrak{m}_{\pi_{m}\left(S_{u}\right)}(v)
\end{array}\right)
$$

This completes the proof.

For example, the number of 2 -partitions $P$ of $\{1, \ldots, 8\}$ whose projection in $\mathbb{Z} / 2 \mathbb{Z}$ is the partition $\pi_{2}(P)=\{\{1,0,0,0\},\{1,1,1,0\}\}$ is equal to 16 , since $\left(\left(\begin{array}{l}4 \\ 3\end{array}\right) \cdot\left(\begin{array}{l}4 \\ 1\end{array}\right)\right) \cdot\left(\left(\begin{array}{c}4-3 \\ 1\end{array}\right) \cdot\left(\begin{array}{c}4-1 \\ 3\end{array}\right)\right)=$ $4 \cdot 4=16$. These 16 partitions are given below.

$$
\begin{array}{llll}
\{\{1,2,4,6\},\{3,5,7,8\}\}, & \{\{1,2,4,8\},\{3,5,7,6\}\}, & \{\{1,2,6,8\},\{3,5,7,4\}\}, \\
\{\{1,4,6,8\},\{3,5,7,2\}\}, & \{\{3,2,4,6\},\{1,5,7,8\}\}, & \{\{3,2,4,8\},\{1,5,7,6\}\}, \\
\{\{3,2,6,8\},\{1,5,7,4\}\}, & \{\{3,4,6,8\},\{1,5,7,2\}\}, & \{\{5,2,4,6\},\{1,3,7,8\}\}, \\
\{\{5,2,4,8\},\{1,3,7,6\}\}, & \{\{5,2,6,8\},\{1,3,7,4\}\}, & \{\{5,4,6,8\},\{1,3,7,2\}\}, \\
\{\{7,2,4,6\},\{1,3,5,8\}\}, & \{\{7,2,4,8\},\{1,3,5,6\}\}, & \{\{7,2,6,8\},\{1,3,5,4\}\}, \\
\{\{7,4,6,8\},\{1,3,5,2\}\} . & &
\end{array}
$$

\section{$4 \mathrm{WS}_{m}(k, l)$ for the modulus $m=2$}

The goal of this section is to prove Theorem 1.3, that is,

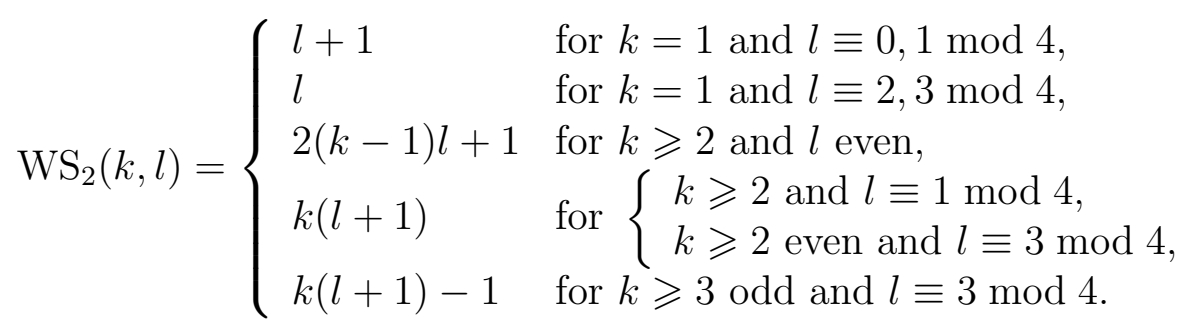

The proof of Theorem 1.3 is based on the following two lemmas.

Lemma 4.1. For any weakly l-sum-free multiset $M$ of $\mathbb{Z} / 2 \mathbb{Z}$, with $|M| \geqslant 1$, we have

1. $\mathfrak{m}_{M}(0) \leqslant l$.

2. $\mathfrak{m}_{M}(1) \leqslant l$ if $l$ is odd.

3. $|M| \leqslant l+1$ if $|M \cap \mathbb{Z} / 2 \mathbb{Z}|=2$. 
Proof.

1. Since $\sum_{i=1}^{l} 0=0$, the multiset $M$ cannot contain more than $l$ terms $0 \in \mathbb{Z} / 2 \mathbb{Z}$.

2. Suppose that $l$ is odd, that is $\pi_{2}(l)=1$. Since $\sum_{i=1}^{l} 1=\pi_{2}(l)=1$, the multiset $M$ cannot contain more than $l$ elements $1 \in \mathbb{Z} / 2 \mathbb{Z}$.

3. Suppose that $M=\left\{0,1, x_{1}, \ldots, x_{l}\right\}$ is a weakly $l$-sum-free multiset of $\mathbb{Z} / 2 \mathbb{Z}$ with cardinality $|M|=l+2$. Since the sum of the $l$ elements $\sum_{i=1}^{l} x_{i}$ is equal to either 0 or 1 , which are both contained in $M \backslash\left\{x_{1}, \ldots, x_{l}\right\}$, we obtain a contradiction.

Remark. For $l$ even, a multiset only constituted by elements $1 \in \mathbb{Z} / 2 \mathbb{Z}$, with any cardinality $|M| \geqslant 1$, is always weakly $l$-sum free in $\mathbb{Z} / 2 \mathbb{Z}$ since $\sum_{i=1}^{l} 1=\pi_{2}(l)=0$.

Lemma 4.2. Let $M=\left\{x_{1}, \ldots, x_{l+1}\right\}$ be a multiset of $\mathbb{Z} / 2 \mathbb{Z}$ with cardinality $|M|=l+1$. Then, $M$ is weakly l-sum-free if and only if $\sum_{i=1}^{l+1} x_{i}=1$.

Proof. First, by definition, the multiset $M$ is weakly $l$-sum-free if and only if, for every $1 \leqslant j \leqslant l+1$, the inequality $\sum_{i=1, i \neq j}^{l+1} x_{i} \neq x_{j}$ holds. Moreover, since

$$
\begin{aligned}
\sum_{\substack{i=1 \\
i \neq j}}^{l+1} x_{i} \neq x_{j} \text { for all } 1 \leqslant j \leqslant l+1 & \Longleftrightarrow \sum_{i=1}^{l+1} x_{i} \neq 2 x_{j}=0 \text { for all } 1 \leqslant j \leqslant l+1 \\
& \Longleftrightarrow \sum_{i=1}^{l+1} x_{i}=1,
\end{aligned}
$$

the result follows.

We are now ready to prove Theorem 1.3, the main result of this section.

\subsection{For $k=1$}

Let $k=1$ and let $l$ be a positive integer. We will prove that

$$
\mathrm{WS}_{2}(1, l)= \begin{cases}l+1 & \text { for } l \equiv 0,1 \bmod 4 \\ l & \text { for } l \equiv 2,3 \bmod 4 .\end{cases}
$$

Claim 4.3. $\mathrm{WS}_{2}(1, l) \in\{l, l+1\}$ for all positive integers $l$.

Proof. Since a multiset of $l$ elements in $\mathbb{Z} / 2 \mathbb{Z}$ is always weakly $l$-sum-free, the inequality $\mathrm{WS}_{2}(1, l) \geqslant l$ holds. Moreover, from Lemma 4.1, we know that a weakly $l$-sum-free multiset $M$ such that $|M \cap \mathbb{Z} / 2 \mathbb{Z}|=2$ has cardinality of at most $l+1$. Therefore $\mathrm{WS}_{2}(1, l) \leqslant l+1$.

Claim 4.4. Let $S=\{1,2, \ldots, l+1\}$. Then, the multiset $\pi_{2}(S)$ is weakly l-sum-free if and only if $l \equiv 0$ or $1 \bmod 4$. 
Proof. By Lemma 4.2, the multiset $\pi_{2}(S)$ is weakly $l$-sum-free if and only if $\sum_{x \in S} x \equiv$ $1 \bmod 2$. Moreover, since

$$
\sum_{x \in S} x=\sum_{x=1}^{l+1} x=\frac{(l+1)(l+2)}{2} \equiv 1 \quad(\bmod 2)
$$

if and only if $l \equiv 0$ or $1 \bmod 4$, the result follows.

\subsection{For $k \geqslant 2$ and $l$ even}

Let $k \geqslant 2$ and $l$ be two positive integers, with $l$ even. We will prove that

$$
\mathrm{WS}_{2}(k, l)=2(k-1) l+1 \text {. }
$$

Claim 4.5. $\mathrm{WS}_{2}(k, l) \leqslant 2(k-1) l+1$ for $k \geqslant 2$ and $l$ even.

Proof. Let $S=\{1,2, \ldots, 2(k-1) l+2\}$. Suppose that there exists a partition $P=$ $\left\{S_{1}, \ldots, S_{k}\right\}$ of the multiset $\pi_{2}(S)$ into $k$ weakly $l$-sum-free multisets of $\mathbb{Z} / 2 \mathbb{Z}$. So the multiplicity function of $\pi_{2}(S)$ is defined by $\mathfrak{m}_{\pi_{2}(S)}(0)=\mathfrak{m}_{\pi_{2}(S)}(1)=(k-1) l+1$. Since $\mathfrak{m}_{S_{i}}(0) \leqslant l$ for every $1 \leqslant i \leqslant k$ by Lemma 4.1 and $\sum_{i=1}^{k} \mathfrak{m}_{S_{i}}(0)=\mathfrak{m}_{\pi_{2}(S)}(0)=l(k-1)+1$, it follows that $\mathfrak{m}_{S_{i}}(0) \geqslant 1$ for every $1 \leqslant i \leqslant k$ by the pigeonhole principle. It follows that $\left|S_{i}\right| \leqslant l+1$ for every $1 \leqslant i \leqslant k$ by Lemma 4.1 again and thus we obtain the following upper bound of the cardinality of $\pi_{2}(S)$,

$$
\left|\pi_{2}(S)\right|=\sum_{i=1}^{k}\left|S_{i}\right| \leqslant k(l+1)
$$

Since

$$
(2(k-1) l+2)-k(l+1)=2 k l-2 l+2-k l-k=k(l-1)-2(l-1)=(k-2)(l-1),
$$

we obtain that $2(k-1) l+2>k(l+1)$ for all $l \geqslant 2$ and $k \geqslant 3$, in contradiction with the previous inequality. For $k=2$, we have $\mathfrak{m}_{\pi_{2}(S)}(0)=\mathfrak{m}_{\pi_{2}(S)}(1)=l+1$ and $\left|S_{1}\right|=\left|S_{2}\right|=l+1$. It follows from Lemma 4.2 that $\sum_{x_{1} \in S_{1}} x_{1}=\sum_{x_{2} \in S_{2}} x_{2}=1$ and the contradiction comes from the following equality

$$
0=1+1=\sum_{x_{1} \in S_{1}} x_{1}+\sum_{x_{2} \in S_{2}} x_{2}=\sum_{x \in \pi_{2}(S)} x=\pi_{2}\left(\sum_{x=1}^{2 l+2} x\right)=\pi_{2}((l+1)(2 l+3)) \stackrel{l \text { even }}{=} 1 .
$$

This completes the proof.

Claim 4.6. $\mathrm{WS}_{2}(k, l) \geqslant 2(k-1) l+1$ for $k \geqslant 2$ and $l$ even.

Proof. Let $S=\{1,2, \ldots, 2(k-1) l+1\}$. We will exhibit a partition $P=\left\{S_{1}, \ldots, S_{k}\right\}$ of $\pi_{2}(S)$ into $k$ weakly $l$-sum-free multisets of $\mathbb{Z} / 2 \mathbb{Z}$. Only the multiplicities of the elements constituting the multisets $S_{i}$ are reported here. 


\begin{tabular}{|c|c|c|c|}
\hline$M$ & $\mathfrak{m}_{M}(0)$ & $\mathfrak{m}_{M}(1)$ & $|M|$ \\
\hline \hline$S_{1}$ & 0 & $(k-1) l+1$ & $(k-1) l+1$ \\
\hline$S_{2}, \ldots, S_{k}$ & $l$ & 0 & $l$ \\
\hline \hline$\pi_{2}(S)$ & $(k-1) l$ & $(k-1) l+1$ & $2(k-1) l+1$ \\
\hline
\end{tabular}

First, $P$ is a partition of $\pi_{2}(S)$ since the multiplicity functions verify that $\sum_{i=1}^{k} \mathfrak{m}_{S_{i}}(0)=$ $\mathfrak{m}_{\pi_{2}(S)}(0)=l(k-1)$ and $\sum_{i=1}^{k} \mathfrak{m}_{S_{i}}(1)=\mathfrak{m}_{\pi_{2}(S)}(1)=l(k-1)+1$. The multiset $S_{1}$ is weakly $l$-sum-free because, as already remarked above, a multiset which is only constituted by elements $1 \in \mathbb{Z} / 2 \mathbb{Z}$ is always weakly $l$-sum-free when $l$ is even. For the other multisets $S_{2}, \ldots, S_{k}$, we already know that multisets containing only $l$ elements are always weakly $l$-sum-free. This completes the proof.

\subsection{For $k \geqslant 2$ and $l$ odd}

Let $k \geqslant 2$ and $l$ be two positive integers, with $l$ odd. We will prove that

$$
\mathrm{WS}_{2}(k, l)= \begin{cases}k(l+1) & \text { for }\left\{\begin{array}{l}
k \text { even and } l \text { odd } \\
k \text { odd and } l \equiv 1 \bmod 4 \\
k(l+1)-1
\end{array}\right. \\
\text { for } k \text { odd and } l \equiv 3 \bmod 4\end{cases}
$$

Claim 4.7. $\mathrm{WS}_{2}(k, l) \leqslant k(l+1)$ for $k \geqslant 1$ and $l$ odd.

Proof. Directly follows from Lemma 4.1.

Claim 4.8. $\mathrm{WS}_{2}(k, l) \leqslant k(l+1)-1$ for $k$ odd and $l \equiv 3 \bmod 4$.

Proof. Let $S=\{1,2, \ldots, k(l+1)\}$. Suppose that there exists a partition $P=\left\{S_{1}, \ldots, S_{k}\right\}$ of $\pi_{2}(S)$ into $k$ weakly $l$-sum-free multisets of $\mathbb{Z} / 2 \mathbb{Z}$. For every $1 \leqslant i \leqslant k$, since $S_{i}$ is a weakly $l$-sum-free multiset and since $l$ is odd, we know from Lemma 4.1 that $\left|S_{i}\right| \leqslant l+1$. Moreover, we have $\sum_{i=1}^{k}\left|S_{i}\right|=\left|\pi_{2}(S)\right|=k(l+1)$. Therefore, for all $1 \leqslant i \leqslant k$, the multiset $S_{i}$ has cardinality of $\left|\pi_{2}\left(S_{i}\right)\right|=l+1$ and thus $\sum_{x \in S_{i}} x=1$ by Lemma 4.2. Since $l \equiv 3 \bmod 4$, this leads to

$$
\pi_{2}(k)=\sum_{i=1}^{k} 1=\sum_{i=1}^{k} \sum_{x \in S_{i}} x=\sum_{x \in \pi_{2}(S)} x=\pi_{2}\left(\sum_{i=1}^{k(l+1)} i\right)=\pi_{2}\left(\frac{l+1}{2} k(k(l+1)+1)\right)=0,
$$

in contradiction with the hypothesis that $k$ is odd. This completes the proof.

Claim 4.9. $\mathrm{WS}_{2}(k, l) \geqslant \mathrm{WS}_{2}(k-2, l)+2(l+1)$ for $k \geqslant 3$ and $l$ odd.

Proof. Let $S=\left\{1,2, \ldots, \mathrm{WS}_{2}(k-2, l)\right\}$ and let $P=\left\{S_{1}, \ldots, S_{k-2}\right\}$ be a partition of $\pi_{2}(S)$ into $k$ weakly $l$-sum-free multisets of $\mathbb{Z} / 2 \mathbb{Z}$. Consider the multisets $S_{k-1}$ and $S_{k}$ of $\mathbb{Z} / 2 \mathbb{Z}$ defined below by their multiplicity functions. 


\begin{tabular}{|c|c|c|c|}
\hline$M$ & $\mathfrak{m}_{M}(0)$ & $\mathfrak{m}_{M}(1)$ & $|M|$ \\
\hline \hline$S_{k-1}$ & $l$ & 1 & $l+1$ \\
\hline$S_{k}$ & 1 & $l$ & $l+1$ \\
\hline \hline$S_{k-1} \cup S_{k}$ & $l+1$ & $l+1$ & $2(l+1)$ \\
\hline
\end{tabular}

Then $P^{\prime}=\left\{S_{1}, \ldots, S_{k}\right\}$ is a partition of the multiset $\pi_{2}\left(S^{\prime}\right)=\pi_{2}\left(\left\{1,2, \ldots, \mathrm{WS}_{2}(k-2, l)+\right.\right.$ $2(l+1)\})$ since $\sum_{i=1}^{k} \mathfrak{m}_{S_{i}}(x)=\mathfrak{m}_{\pi_{2}(S)}(x)+(l+1)=\mathfrak{m}_{\pi_{2}\left(S^{\prime}\right)}(x)$ for all $x \in \mathbb{Z} / 2 \mathbb{Z}$. Moreover, since $l$ is odd, it follows that $\sum_{x \in S_{k-1}} x=\sum_{x \in S_{k}}=1$ and thus the multisets $S_{k-1}$ and $S_{k}$ are weakly $l$-sum-free in $\mathbb{Z} / 2 \mathbb{Z}$ by Lemma 4.2 . Therefore $P^{\prime}$ is a partition of $\pi_{2}\left(S^{\prime}\right)$ into $k$ weakly $l$-sum-free multisets.

Claim 4.10. $\mathrm{WS}_{2}(2, l) \geqslant 2(l+1)$ for $l$ odd.

Proof. Let $S=\{1,2, \ldots, 2(l+1)\}$. As already seen in the proof of Claim 4.9, the following partition $P=\left\{S_{1}, S_{2}\right\}$ of $\pi_{2}(S)$ is weakly $l$-sum-free.

\begin{tabular}{|c|c|c|c|}
\hline$M$ & $\mathfrak{m}_{M}(0)$ & $\mathfrak{m}_{M}(1)$ & $|M|$ \\
\hline \hline$S_{1}$ & $l$ & 1 & $l+1$ \\
\hline$S_{2}$ & 1 & $l$ & $l+1$ \\
\hline \hline$S_{1} \cup S_{2}$ & $l+1$ & $l+1$ & $2(l+1)$ \\
\hline
\end{tabular}

This concludes the proof.

We are now ready to prove the formula for all $k \geqslant 2$ and $l$ odd. We distinguish different cases depending on the parity of $k$ and the residue class of $l$ modulo 4 .

Case 1: for $k \geqslant 2$ odd and $l \equiv 1 \bmod 4$.

First, we know that $\mathrm{WS}_{2}(1, l)=l+1$ from Subsection 4.1. By applying $(k-1) / 2$ times the inequality of Claim 4.9, we obtain that

$$
\mathrm{WS}_{2}(k, l) \geqslant \mathrm{WS}_{2}(k-2, l)+2(l+1) \geqslant \cdots \geqslant \mathrm{WS}_{2}(1, l)+(k-1)(l+1)=k(l+1) .
$$

Finally, since $\mathrm{WS}_{2}(k, l) \leqslant k(l+1)$ by Claim 4.7 , it follows that $\mathrm{WS}_{2}(k, l)=k(l+1)$ in this case.

Case 2: for $k \geqslant 2$ odd and $l \equiv 3 \bmod 4$.

First, we know that $\mathrm{WS}_{2}(1, l)=l$ from Subsection 4.1. By applying $(k-1) / 2$ times the inequality of Claim 4.9, we obtain that

$$
\mathrm{WS}_{2}(k, l) \geqslant \mathrm{WS}_{2}(k-2, l)+2(l+1) \geqslant \cdots \geqslant \mathrm{WS}_{2}(1, l)+(k-1)(l+1)=k(l+1)-1 .
$$

Finally, since $\mathrm{WS}_{2}(k, l) \leqslant k(l+1)-1$ by Claim 4.8 , it follows that $\mathrm{WS}_{2}(k, l)=k(l+1)-1$ in this case.

Case 3: for $k \geqslant 2$ even and $l$ odd.

First, we know that $\mathrm{WS}_{2}(2, l) \geqslant 2(l+1)$ from Claim 4.10. By applying $k / 2-1$ times the inequality of Claim 4.9, we obtain that

$$
\mathrm{WS}_{2}(k, l) \geqslant \mathrm{WS}_{2}(k-2, l)+2(l+1) \geqslant \cdots \geqslant \mathrm{WS}_{2}(2, l)+(k-2)(l+1) \geqslant k(l+1),
$$


Finally, since $\mathrm{WS}_{2}(k, l) \leqslant k(l+1)$ by Claim 4.7 , it follows that $\mathrm{WS}_{2}(k, l)=k(l+1)$ in this case.

This concludes the proof of Theorem 1.3.

\section{$5 \quad \mathrm{WS}_{m}(k, l)$ for the modulus $m=3$}

The goal of this section is to prove Theorem 1.4, that is,

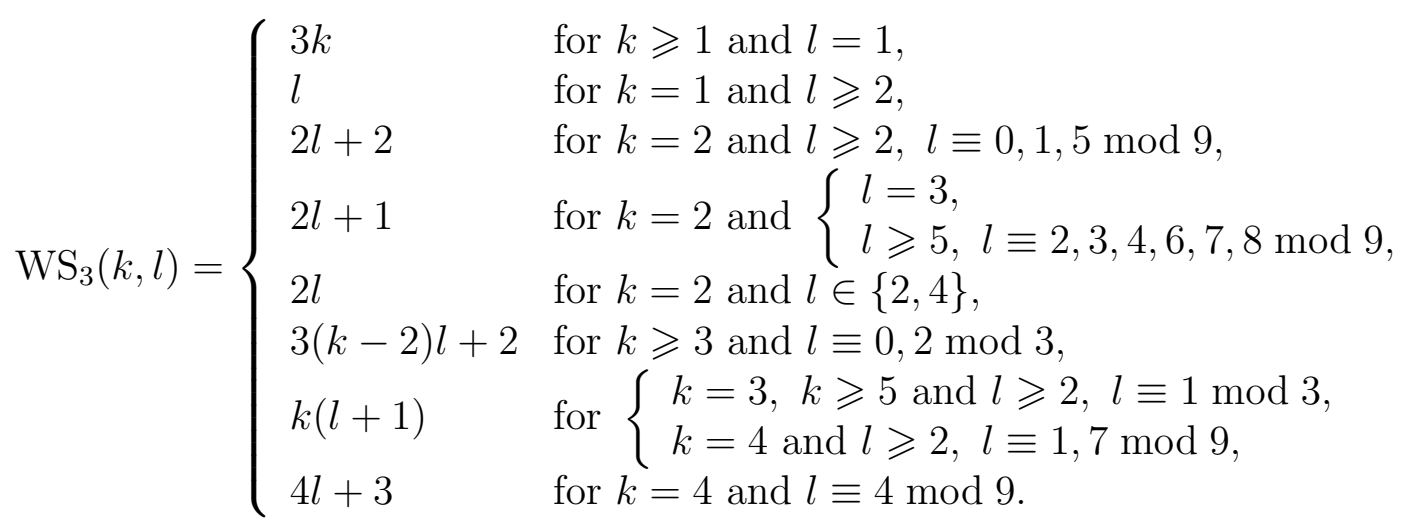

The proof of Theorem 1.4 is based upon the following two lemmas.

Lemma 5.1. For any weakly $l$-sum-free multiset $M$ of $\mathbb{Z} / 3 \mathbb{Z}$, with $|M| \geqslant 1$ and $l \geqslant 2$, we have

1. $\mathfrak{m}_{M}(0) \leqslant l$.

2. $\mathfrak{m}_{M}(1) \leqslant l$ and $\mathfrak{m}_{M}(2) \leqslant l$ if $l \equiv 1 \bmod 3$.

3. $|M| \leqslant l+1$ if $|M \cap \mathbb{Z} / 3 \mathbb{Z}|=2$.

4. $|M| \leqslant l$ if $|M \cap \mathbb{Z} / 3 \mathbb{Z}|=3$.

Proof.

1. Since $\sum_{i=1}^{l} 0=0$, the multiset $M$ cannot contain more than $l$ terms $0 \in \mathbb{Z} / 3 \mathbb{Z}$.

2. Suppose that $l \equiv 1 \bmod 3$. Since $\sum_{i=1}^{l} 1=\pi_{3}(l)=1$ and $\sum_{i=1}^{l} 2=\pi_{3}(2 l)=2$, the multiset $M$ can contain neither more than $l$ elements $1 \in \mathbb{Z} / 3 \mathbb{Z}$ nor more than $l$ elements $2 \in \mathbb{Z} / 3 \mathbb{Z}$.

3. Supppose that $|M|=l+2$ with $M \cap \mathbb{Z} / 3 \mathbb{Z}=\{x, y\}$. Since $l+2 \geqslant 4$, we can suppose that $\mathfrak{m}_{M}(x) \geqslant 2$. Moreover, either $x=y+1$ or $x=y+2$. Without loss of generality, suppose that $x=y+1$ and denote $M=\left\{x, x, y, x_{1}, x_{2}, \ldots, x_{l-1}\right\}$. Then,

$$
\text { - } x+\sum_{i=1}^{l-1} x_{i}=x \text { if } \sum_{i=1}^{l-1} x_{i}=0,
$$


- $y+\sum_{i=1}^{l-1} x_{i}=x$ if $\sum_{i=1}^{l-1} x_{i}=1$,

- $x+\sum_{i=1}^{l-1} x_{i}=y$ if $\sum_{i=1}^{l-1} x_{i}=2$.

Therefore $M$ is not weakly $l$-sum-free in $\mathbb{Z} / 3 \mathbb{Z}$.

4. Suppose that $|M|=l+1$ with $M \cap \mathbb{Z} / 3 \mathbb{Z}=\mathbb{Z} / 3 \mathbb{Z}$. Denote $M=\left\{0,1,2, x_{1}, \ldots, x_{l-2}\right\}$. Then,

- $\sum_{i=1}^{l-2} x_{i}+1+2=0$ if $\sum_{i=1}^{l-2} x_{i}=0$,

- $\sum_{i=1}^{l-2} x_{i}+1+0=2$ if $\sum_{i=1}^{l-2} x_{i}=1$,

- $\sum_{i=1}^{l-2} x_{i}+2+0=1$ if $\sum_{i=1}^{l-2} x_{i}=2$.

Therefore $M$ is not weakly $l$-sum-free in $\mathbb{Z} / 3 \mathbb{Z}$.

Lemma 5.2. Let $l \geqslant 2$ and let $M=\left\{x_{1}, \ldots, x_{l+1}\right\}$ be a multiset of $\mathbb{Z} / 3 \mathbb{Z}$ with $|M|=l+1$ and $|M \cap \mathbb{Z} / 3 \mathbb{Z}|=2$. Let $x$ be the element of $\mathbb{Z} / 3 \mathbb{Z}$ such that $x \notin M$. Then, the multiset $M$ is weakly l-sum-free if and only if $\sum_{i=1}^{l+1} x_{i}=2 x$.

Proof. First, by definition, the multiset $M$ is weakly $l$-sum-free if and only if, for every $1 \leqslant j \leqslant l+1$, the inequality $\sum_{i=1, i \neq j}^{l+1} x_{i} \neq x_{j}$ holds. Moreover, since

$$
\begin{aligned}
\sum_{\substack{i=1 \\
i \neq j}}^{l+1} x_{i} \neq x_{j} \text { for all } 1 \leqslant j \leqslant l+1 & \Longleftrightarrow \sum_{i=1}^{l+1} x_{i} \neq 2 x_{j} \text { for all } 1 \leqslant j \leqslant l+1 \\
& \Longleftrightarrow \sum_{i=1}^{l+1} x_{i}=2 x,
\end{aligned}
$$

the result follows.

We are now ready to prove Theorem 1.4, the main result of this section.

\subsection{For $k=1$ and $l=1$}

Claim 5.3. $\mathrm{WS}_{3}(k, 1)=3 k$ for $k \geqslant 1$.

Proof. Obviously, the inequality $\mathrm{WS}_{3}(k, 1) \leqslant 3 k$ holds because a weakly 1 -sum-free multiset of $\mathbb{Z} / 3 \mathbb{Z}$ cannot contain more than once each element of $\mathbb{Z} / 3 \mathbb{Z}$. Let $S=\{1,2, \ldots, 3 k\}$. The partition $P=\left\{S_{1}, \ldots, S_{k}\right\}$, where $S_{i}=\{0,1,2\}$ for all $1 \leqslant i \leqslant k$, is a weakly 1 -sum-free partition of $\pi_{3}(S)$ and thus $\mathrm{WS}_{3}(k, 1) \geqslant 3 k$. This completes the proof.

Claim 5.4. $\mathrm{WS}_{3}(1, l)=l$ for $l \geqslant 2$.

Proof. First, since a multiset of $\mathbb{Z} / 3 \mathbb{Z}$ constituted by only $l$ terms is always weakly $l$-sumfree, it follows that $\mathrm{WS}_{3}(1, l) \geqslant l$. Moreover, by Lemma 5.1 , a weakly $l$-sum-free multiset $M$ such that $|M \cap \mathbb{Z} / 3 \mathbb{Z}|=3$ has cardinality of at most $l$. Therefore $\operatorname{WS}_{3}(1, l) \leqslant l$. This completes the proof. 


\subsection{For $k=2$}

Let $k=2$ and let $l \geqslant 2$ be a positive integer. We will prove that

$$
\mathrm{WS}_{3}(2, l)= \begin{cases}2 l+2 & \text { for } l \geqslant 2, l \equiv 0,1,5 \bmod 9 \\
2 l+1 & \text { for }\left\{\begin{array}{l}
l=3 \\
l \geqslant 5, l
\end{array}, \quad \text { for } l \in\{2,4\}\right.\end{cases}
$$

Claim 5.5. Let $S=\{1,2, \ldots, n\}$ where $n \geqslant 3$. Suppose that there exists a partition $P=\left\{S_{1}, S_{2}\right\}$ of $\pi_{3}(S)$ into 2 weakly l-sum-free multisets. Then, the multisets $S_{1}$ and $S_{2}$ both have cardinality of at most $l+1$.

Proof. Suppose, without loss of generality, that $\left|S_{1}\right| \geqslant l+2$. First, we deduce from Lemma 5.1 that $\left|S_{1} \cap \mathbb{Z} / 3 \mathbb{Z}\right|=1$. Let $x \in \mathbb{Z} / 3 \mathbb{Z}$ such that $S_{1} \cap \mathbb{Z} / 3 \mathbb{Z}=\{x\}$. Obviously, we have $\mathfrak{m}_{\pi_{3}(S)}(x) \leqslant \mathfrak{m}_{\pi_{3}(S)}(x+1)+\mathfrak{m}_{\pi_{3}(S)}(x+2)$. This leads to the inequality

$\left|S_{1}\right|=\mathfrak{m}_{S_{1}}(x) \leqslant \mathfrak{m}_{S_{1} \cup S_{2}}(x) \leqslant \mathfrak{m}_{S_{1} \cup S_{2}}(x+1)+\mathfrak{m}_{S_{1} \cup S_{2}}(x+2)=\mathfrak{m}_{S_{2}}(x+1)+\mathfrak{m}_{S_{2}}(x+2) \leqslant\left|S_{2}\right|$.

It follows that $\left|S_{2}\right| \geqslant l+2$ and thus $\left|S_{2} \cap \mathbb{Z} / 3 \mathbb{Z}\right|=1$, by Lemma 5.1 again, in contradiction with $\left|\pi_{3}(S) \cap \mathbb{Z} / 3 \mathbb{Z}\right|=3$.

Claim 5.6. $\mathrm{WS}_{3}(2, l) \in\{2 l, 2 l+1,2 l+2\}$ for all positive integers $l \geqslant 2$.

Proof. Since a multiset of $l$ elements in $\mathbb{Z} / 3 \mathbb{Z}$ is always weakly $l$-sum-free, the inequality $\mathrm{WS}_{3}(2, l) \geqslant 2 l$ holds. Moreover, from Claim 5.5, we know that each multiset of a weakly $l$-sum-free 2-partition has cardinality of at most $l+1$. Therefore $\mathrm{WS}_{2}(2, l) \leqslant 2(l+1)$.

Claim 5.7. $\mathrm{WS}_{3}(2, l) \geqslant 2 l+2$ if and only if $l \equiv 0,1,5 \bmod 9$.

Proof. Let $S=\{1,2, \ldots, 2 l+2\}$. Suppose that there exists a partition $P=\left\{S_{1}, S_{2}\right\}$ of $\pi_{3}(S)$ into 2 weakly $l$-sum-free multisets of $\mathbb{Z} / 3 \mathbb{Z}$. Since $\left|S_{1}\right|=\left|S_{2}\right|=l+1$ by Claim 5.5 , it follows from Lemma 5.1 that $\left|S_{1} \cap \mathbb{Z} / 3 \mathbb{Z}\right|=\left|S_{2} \cap \mathbb{Z} / 3 \mathbb{Z}\right|=2$.

Case 1: if $0 \in S_{1}$ and $0 \in S_{2}$.

Without lost of generality, suppose that $S_{1} \cap \mathbb{Z} / 3 \mathbb{Z}=\{0,1\}$ and $S_{2} \cap \mathbb{Z} / 3 \mathbb{Z}=\{0,2\}$. By Lemma 5.2, we know that the multisets $S_{1}$ and $S_{2}$ are weakly $l$-sum-free if and only if $\sum_{x \in S_{1}} x=2.2=1$ and $\sum_{x \in S_{2}} x=2.1=2$. It follows that $\mathfrak{m}_{S_{1}}(1) \equiv 1 \bmod 3$ and $\mathfrak{m}_{S_{2}}(2) \equiv 1 \bmod 3$. This implies that

$$
\mathfrak{m}_{\pi_{3}(S)}(1)=\mathfrak{m}_{S_{1}}(1) \equiv 1 \equiv \mathfrak{m}_{S_{2}}(2)=\mathfrak{m}_{\pi_{3}(S)}(2) \quad(\bmod 3) .
$$

Since $S$ is the set of the first $2 l+2$ positive integers, it follows that $\mathfrak{m}_{\pi_{3}(S)}(1)=\mathfrak{m}_{\pi_{3}(S)}(2)$ or $\mathfrak{m}_{\pi_{3}(S)}(1)=\mathfrak{m}_{\pi_{3}(S)}(2)+1$. Therefore $\mathfrak{m}_{\pi_{3}(S)}(1)=\mathfrak{m}_{\pi_{3}(S)}(2) \equiv 1 \bmod 3$. Moreover, by definition of the set $S$ again, either $\mathfrak{m}_{\pi_{3}(S)}(0)=\mathfrak{m}_{\pi_{3}(S)}(1)$ or $\mathfrak{m}_{\pi_{3}(S)}(0)=\mathfrak{m}_{\pi_{3}(S)}(1)-1$.

Case 1.1: if $\mathfrak{m}_{\pi_{3}(S)}(0)=\mathfrak{m}_{\pi_{3}(S)}(1)=\mathfrak{m}_{\pi_{3}(S)}(2) \equiv 1 \bmod 3$.

Then, $2 l+2=\mathfrak{m}_{\pi_{3}(S)}(0)+\mathfrak{m}_{\pi_{3}(S)}(1)+\mathfrak{m}_{\pi_{3}(S)}(2) \equiv 3 \bmod 9$ and thus $l \equiv 5 \bmod 9$. In this case, $l \equiv 5 \bmod 9$, we can verify with Lemma 5.2 that the following 2 -partition of $\pi_{3}(S)$ is weakly $l$-sum-free. 


\begin{tabular}{|c|c|c|c|c|}
\hline$M$ & $\mathfrak{m}_{M}(0)$ & $\mathfrak{m}_{M}(1)$ & $\mathfrak{m}_{M}(2)$ & $|M|$ \\
\hline \hline$S_{1}$ & $(l+1) / 3$ & $2(l+1) / 3$ & 0 & $l+1$ \\
\hline$S_{2}$ & $(l+1) / 3$ & 0 & $2(l+1) / 3$ & $l+1$ \\
\hline \hline$\pi_{3}(S)$ & $2(l+2) / 3$ & $2(l+2) / 3$ & $2(l+2) / 3$ & $2 l+2$ \\
\hline
\end{tabular}

This proves that $\mathrm{WS}_{3}(2, l) \geqslant 2 l+2$ for all $l \equiv 5 \bmod 9$.

Case 1.2: if $\mathfrak{m}_{\pi_{3}(S)}(0)+1=\mathfrak{m}_{\pi_{3}(S)}(1)=\mathfrak{m}_{\pi_{3}(S)}(2) \equiv 1 \bmod 3$.

Then, $2 l+2=\mathfrak{m}_{\pi_{3}(S)}(0)+\mathfrak{m}_{\pi_{3}(S)}(1)+\mathfrak{m}_{\pi_{3}(S)}(2) \equiv 2 \bmod 9$ and thus $l \equiv 0 \bmod 9$. We can verify with Lemma 5.2 that the following 2-partition of $\pi_{3}(S)$ is weakly $l$-sum-free in this case.

\begin{tabular}{|c|c|c|c|c|}
\hline$M$ & $\mathfrak{m}_{M}(0)$ & $\mathfrak{m}_{M}(1)$ & $\mathfrak{m}_{M}(2)$ & $|M|$ \\
\hline \hline$S_{1}$ & $l / 3$ & $2 l / 3+1$ & 0 & $l+1$ \\
\hline$S_{2}$ & $l / 3$ & 0 & $2 l / 3+1$ & $l+1$ \\
\hline \hline$\pi_{3}(S)$ & $2 l / 3$ & $2 l / 3+1$ & $2 l / 3+1$ & $2 l+2$ \\
\hline
\end{tabular}

This proves that $\mathrm{WS}_{3}(2, l) \geqslant 2 l+2$ for all $l \equiv 0 \bmod 9$.

Case 2: if only one of the multisets $S_{1}$ and $S_{2}$ contains elements $0 \in \mathbb{Z} / 3 \mathbb{Z}$.

Without loss of generality, we can suppose that this is $S_{1}$. Then, we have $S_{1} \cap \mathbb{Z} / 3 \mathbb{Z}=$ $\{0, a\}$ and $S_{2} \cap \mathbb{Z} / 3 \mathbb{Z}=\{a, 2 a\}$ with $a \in\{1,2\} \subset \mathbb{Z} / 3 \mathbb{Z}$. By Lemma 5.2 , we know that the multisets $S_{1}$ and $S_{2}$ are weakly $l$-sum-free if and only if $\sum_{x \in S_{1}} x=2.2 a=a$ and $\sum_{x \in S_{2}} x=2.0=0$. It follows that $\mathfrak{m}_{S_{1}}(a) \equiv 1 \bmod 3$ and $\mathfrak{m}_{S_{2}}(a) \equiv \mathfrak{m}_{S_{2}}(2 a) \bmod 3$. This implies that

$$
\mathfrak{m}_{\pi_{3}(S)}(a)=\mathfrak{m}_{S_{1}}(a)+\mathfrak{m}_{S_{2}}(a) \equiv 1+\mathfrak{m}_{S_{2}}(2 a)=1+\mathfrak{m}_{\pi_{3}(S)}(2 a) \quad(\bmod 3)
$$

Since $\mathfrak{m}_{\pi_{3}(S)}(1)=\mathfrak{m}_{\pi_{3}(S)}(2)$ or $\mathfrak{m}_{\pi_{3}(S)}(1)=\mathfrak{m}_{\pi_{3}(S)}(2)+1$ by definition of the set $S$, we deduce that $a=1$ and $\mathfrak{m}_{\pi_{3}(S)}(1)=\mathfrak{m}_{\pi_{3}(S)}(2)+1$. Moreover, by definition of the set $S$ again, we have

$$
\mathfrak{m}_{\pi_{3}(S)}(0)=\mathfrak{m}_{\pi_{3}(S)}(2)=\mathfrak{m}_{\pi_{3}(S)}(1)-1 .
$$

Then $2 l+2=\mathfrak{m}_{\pi_{3}(S)}(0)+\mathfrak{m}_{\pi_{3}(S)}(1)+\mathfrak{m}_{\pi_{3}(S)}(2)=3 \mathfrak{m}_{\pi_{3}(S)}(0)+1 \equiv 1 \bmod 3$ and thus $l \equiv 1 \bmod 3$. It follows that $2 \equiv l+1=\mathfrak{m}_{S_{2}}(1)+\mathfrak{m}_{S_{2}}(2) \equiv 2 \mathfrak{m}_{S_{2}}(2)=2 \cdot \mathfrak{m}_{\pi_{3}(S)}(2) \bmod 3$. Therefore

$$
\mathfrak{m}_{\pi_{3}(S)}(0)=\mathfrak{m}_{\pi_{3}(S)}(2)=\mathfrak{m}_{\pi_{3}(S)}(1)-1 \equiv 1 \quad(\bmod 3) .
$$

Then $2 l+2=\mathfrak{m}_{\pi_{3}(S)}(0)+\mathfrak{m}_{\pi_{3}(S)}(1)+\mathfrak{m}_{\pi_{3}(S)}(2) \equiv 4 \bmod 9$ and thus $l \equiv 1 \bmod 9$. We can verify with Lemma 5.2 that the following 2-partition of $\pi_{3}(S)$ is weakly $l$-sum-free in this case.

\begin{tabular}{|c|c|c|c|c|}
\hline$M$ & $\mathfrak{m}_{M}(0)$ & $\mathfrak{m}_{M}(1)$ & $\mathfrak{m}_{M}(2)$ & $|M|$ \\
\hline \hline$S_{1}$ & $(2 l+1) / 3$ & $(l+2) / 3$ & 0 & $l+1$ \\
\hline$S_{2}$ & 0 & $(l+2) / 3$ & $(2 l+1) / 3$ & $l+1$ \\
\hline \hline$\pi_{3}(S)$ & $(2 l+1) / 3$ & $(2 l+4) / 3$ & $(2 l+1) / 3$ & $2 l+2$ \\
\hline
\end{tabular}


This proves that $\mathrm{WS}_{3}(2, l) \geqslant 2 l+2$ for all $l \geqslant 2, l \equiv 1 \bmod 9$.

This completes the proof that $\mathrm{WS}_{3}(2, l) \geqslant 2 l+2$ if and only if $l \equiv 0,1,5 \bmod 9$.

Claim 5.8. $\mathrm{WS}_{3}(2, l) \geqslant 2 l+1$ for $l \geqslant 9$.

Proof. Let $S=\{1,2, \ldots, 2 l+1\}$. We will prove that there exists a partition of $\pi_{3}(S)$ into 2 weakly $l$-sum-free multisets. Consider the euclidean division of $l \geqslant 9$ by $3: l=3 q+r$ where $q \geqslant 3$ and $r \in\{0,1,2\}$. Then, $l+1=3 q+r+1$ and $2 l+1=6 q+2 r+1$ and $\pi_{3}(S)$ is a multiset of $\mathbb{Z} / 3 \mathbb{Z}$ whose multiplicity function verifies that

$$
\mathfrak{m}_{\pi_{3}(S)}(1) \geqslant 2 q+1, \quad \mathfrak{m}_{\pi_{3}(S)}(2) \geqslant 2 q \quad \text { and } \quad \mathfrak{m}_{\pi_{3}(S)}(0) \geqslant 2 q
$$

First, we prove that there exists a submultiset $S_{1}$ with $\left|S_{1}\right|=l+1$ which is weakly $l$-sumfree. The multiset $S_{1}$ is defined below by its multiplicity function, distinguishing different cases depending on the residue class of $q$ modulo 3 .

\begin{tabular}{|c|c|c|c|}
\hline$\pi_{3}(q)$ & $\mathfrak{m}_{S_{1}}(0)$ & $\mathfrak{m}_{S_{1}}(1)$ & $\mathfrak{m}_{S_{1}}(2)$ \\
\hline \hline 0 & $q+r$ & $2 q+1$ & 0 \\
\hline 1 & $q+r+2$ & $2 q-1$ & 0 \\
\hline 2 & $q+r+1$ & $2 q$ & 0 \\
\hline
\end{tabular}

Case 1: for $q \equiv 0 \bmod 3$.

The multiset $S_{1}$ is well defined because $\mathfrak{m}_{S_{1}}(0)=q+r \leqslant 2 q \leqslant \mathfrak{m}_{\pi_{3}(S)}(0)$ and $\mathfrak{m}_{S_{1}}(1)=$ $\mathfrak{m}_{\pi_{3}(S)}(1)$. Moreover, it is weakly $l$-sum-free by Lemma 5.2 since

$$
\sum_{x \in S_{1}} x=(q+r) \cdot 0+(2 q+1) \cdot 1=\pi_{3}(2 q+1)=1=2.2
$$

Case 2: for $q \equiv 1 \bmod 3$.

Remark that $q \geqslant 4$ in this case. The multiset $S_{1}$ is well defined because $\mathfrak{m}_{S_{1}}(0)=$ $q+r+2 \leqslant 2 q \leqslant \mathfrak{m}_{\pi_{3}(S)}(0)$ and $\mathfrak{m}_{S_{1}}(1)=2 q-1 \leqslant 2 q+1 \leqslant \mathfrak{m}_{\pi_{3}(S)}(1)$. Moreover, it is weakly $l$-sum-free by Lemma 5.2 since

$$
\sum_{x \in S_{1}} x=(q+r+2) .0+(2 q-1) \cdot 1=\pi_{3}(2 q-1)=1=2.2 .
$$

Case 3: for $q \equiv 2 \bmod 3$.

The multiset $S_{1}$ is well defined because $\mathfrak{m}_{S_{1}}(0)=q+r+1 \leqslant 2 q \leqslant \mathfrak{m}_{\pi_{3}(S)}(0)$ and $\mathfrak{m}_{S_{1}}(1)=$ $2 q \leqslant 2 q+1 \mathfrak{m}_{\pi_{3}(S)}(1)$. Moreover, it is weakly $l$-sum-free by Lemma 5.2 since

$$
\sum_{x \in S_{1}} x=(q+r+1) \cdot 0+(2 q) \cdot 1=\pi_{3}(2 q)=1=2.2 .
$$

Finally, if $S_{2}$ is the multiset, with cardinality $l$, constituted by all the other elements of $\pi_{3}(S) \backslash S_{1}$, then $S_{2}$ is clearly weakly $l$-sum-free and we have obtained a weakly $l$-sum-free 2-partition of $\pi_{3}(S)$. 
Thus, by Claim 5.6, Claim 5.7 and Claim 5.8, we have already proved that

$$
\mathrm{WS}_{3}(2, l)=\left\{\begin{array}{l}
2 l+2 \text { for } l \equiv 0,1,5 \bmod 9 \\
2 l+1 \text { for } l \geqslant 9, l \equiv 2,3,4,6,7,8 \bmod 9 .
\end{array}\right.
$$

It remains to settle the cases $l \in\{2,3,4,6,7,8\}$.

Claim 5.9. $\mathrm{WS}_{3}(2,2) \leqslant 4$.

Proof. Let $S=\{1,2,3,4,5\}$. Then $\pi_{3}(\{1,2,3,4,5\})=\{0,1,1,2,2\}$. In any 2-partition of $\pi_{3}(S)$ there is one multiset with cardinality of at least 3 . Since the submultisets $\{0,1,1\}$, $\{0,1,2\},\{0,2,2\},\{1,1,2\}$ and $\{2,2,1\}$ are not weakly 2 -sum-free in $\mathbb{Z} / 3 \mathbb{Z}$, it follows that there is no weakly 2 -sum-free 2 -partition of $\pi_{3}(S)$. Therefore $\mathrm{WS}_{3}(2,2) \leqslant 4$.

So, from Claim 5.6 and Claim 5.9, we obtain that $\mathrm{WS}_{3}(2,2)=4$.

Claim 5.10. $\mathrm{WS}_{3}(2,4) \leqslant 8$.

Proof. Let $S=\{1,2,3,4,5,6,7,8,9\}$. Then $\pi_{3}(S)=\{0,0,0,1,1,1,2,2,2\}$. In any 2partition of $\pi_{3}(S)$ there is one multiset with cardinality of at least 5 . Since a weakly 4 -sum-free multiset with cardinality of 5 contains exactly 2 different terms of $\mathbb{Z} / 3 \mathbb{Z}$ by Lemma 5.1 and since the submultisets

$$
\{0,0,1,1,1\},\{0,0,0,1,1\},\{1,1,1,2,2\},\{1,1,2,2,2\},\{0,0,2,2,2\},\{0,0,0,2,2\}
$$

are not weakly 4 -sum-free in $\mathbb{Z} / 3 \mathbb{Z}$ by Lemma 5.2 , it follows that there is no weakly 4-sum-free 2-partition of $\pi_{3}(S)$. Therefore $\mathrm{WS}_{3}(2,4) \leqslant 8$.

Thus, from Claim 5.6 and Claim 5.10, we obtain that $\mathrm{WS}_{3}(2,4)=8$.

Claim 5.11. $\mathrm{WS}_{3}(2, l) \geqslant 2 l+1$ for $l \in\{3,6,7,8\}$.

Proof. Let $S=\{1,2, \ldots, 2 l+1\}$. We can verify by using Lemma 5.2 that the following

\begin{tabular}{|c|c|c|c|c|c|}
\hline \multirow{4}{*}{$l=3:$} & $M$ & $\mathfrak{m}_{M}(0)$ & $\mathfrak{m}_{M}(1)$ & $\mathfrak{m}_{M}(2)$ & $\mid M$ \\
\hline & $\overline{S_{1}}$ & 0 & 2 & 2 & 4 \\
\hline & $S_{2}$ & 2 & 1 & 0 & 3 \\
\hline & $\pi_{3}(S)$ & 2 & 3 & 2 & 7 \\
\hline
\end{tabular}
partition $P=\left\{S_{1}, S_{2}\right\}$ of $\pi_{3}(S)$ is weakly l-sum-free.

\begin{tabular}{|c|c|c|c|c|c|}
\hline \multirow{4}{*}{$l=6:$} & $M$ & $\mathfrak{m}_{M}(0)$ & $\mathfrak{m}_{M}(1)$ & $\mathfrak{m}_{M}(2)$ & $M$ \\
\hline & $S_{1}$ & 3 & 4 & 0 & 7 \\
\hline & $S_{2}$ & 1 & 1 & 4 & 6 \\
\hline & $\pi_{3}(S)$ & 4 & 5 & 4 & 13 \\
\hline
\end{tabular}

\begin{tabular}{c|c|c|c|c|c|}
\cline { 2 - 6 }$l=7:$ & $M$ & $\mathfrak{m}_{M}(0)$ & $\mathfrak{m}_{M}(1)$ & $\mathfrak{m}_{M}(2)$ & $|M|$ \\
\cline { 2 - 6 } & $S_{1}$ & 4 & 4 & 0 & 8 \\
\cline { 2 - 6 } & $S_{2}$ & 1 & 1 & 5 & 7 \\
\hline \hline$\pi_{3}(S)$ & 5 & 5 & 5 & 15 \\
\hline
\end{tabular}




\begin{tabular}{|c|c|c|c|c|c|}
\hline \multirow{4}{*}{$l=8:$} & $M$ & $\mathfrak{m}_{M}(0)$ & $\mathfrak{m}_{M}(1)$ & $\mathfrak{m}_{M}(2)$ & $M$ \\
\hline & $S_{1}$ & 5 & 4 & 0 & 9 \\
\hline & $S_{2}$ & 0 & 2 & 6 & 8 \\
\hline & $\pi_{3}(S)$ & 5 & 6 & 6 & 17 \\
\hline
\end{tabular}

Finally, by Claim 5.7 and Claim 5.11, we deduce that $\mathrm{WS}_{3}(2, l)=2 l+1$ for $l \in\{3,6,7,8\}$. This concludes the proof of Theorem 1.4 in this case.

\subsection{For $k \geqslant 3$ and $l \equiv 0,2 \bmod 3$}

Let $k \geqslant 3$ and $l$ be two positive integers, with $l \equiv 0$ or $2 \bmod 3$. We will prove that

$$
\mathrm{WS}_{3}(k, l)=3(k-2) l+2 .
$$

Claim 5.12. $\mathrm{WS}_{3}(k, l) \leqslant 3(k-2) l+2$ for $k \geqslant 3$ and $l \equiv 0,2 \bmod 3$.

Proof. Let $S=\{1,2, \ldots, 3(k-2) l+3\}$. Suppose that there exists a partition $P=$ $\left\{S_{1}, \ldots, S_{k}\right\}$ of $\pi_{3}(S)$ into $k$ weakly $l$-sum-free multisets in $\mathbb{Z} / 3 \mathbb{Z}$.

First, we prove that $\left|S_{i}\right| \leqslant l+1$, for all $1 \leqslant i \leqslant k$. From the definition of $\pi_{3}(S)$, we know that $\mathfrak{m}_{\pi_{3}(S)}(x)=(k-2) l+1$, for all $x \in \mathbb{Z} / 3 \mathbb{Z}$. Since $\mathfrak{m}_{S_{i}}(0) \leqslant l$, for all $1 \leqslant i \leqslant k$, by Lemma 5.1 and $\sum_{i=1}^{k} \mathfrak{m}_{S_{i}}(0)=\mathfrak{m}_{\pi_{3}(S)}(0)=(k-2) l+1$, it follows from the pigeonhole principle that there are at least $k-1$ multisets $S_{i}$ containing at least one element $0 \in \mathbb{Z} / 3 \mathbb{Z}$. Moreover, by Lemma 5.1 again, a weakly $l$-sum-free multiset $S_{i}$ for which $\mathfrak{m}_{\pi_{3}\left(S_{i}\right)}(0) \geqslant 1$ has cardinality of at most $l+1$. Therefore, if the number of multisets $S_{i}$ for which $\mathfrak{m}_{S_{i}}(0) \geqslant 1$ is $k$, we have $\left|S_{i}\right| \leqslant l+1$, for all $1 \leqslant i \leqslant k$. In the other case, if the number of multisets $S_{i}$ for which $\mathfrak{m}_{S_{i}}(0) \geqslant 1$ is exactly $k-1$, we can also prove that the $k$ th multiset has cardinality of at most $l+1$. Indeed, if we suppose that $\mathfrak{m}_{S_{i}}(0) \geqslant 1$, for all $1 \leqslant i \leqslant k-1$, and $\mathfrak{m}_{S_{k}}(0)=0$ with $\left|S_{k}\right| \geqslant l+2$, then $S_{k} \cap \mathbb{Z} / 3 \mathbb{Z}=\{a\}$ with $a \in\{1,2\} \subset \mathbb{Z} / 3 \mathbb{Z}$ by Lemma 5.1. Thus, since $l+2=\mathfrak{m}_{\pi_{3}\left(S_{k}\right)}(a) \leqslant \mathfrak{m}_{\pi_{3}(S)}(a)=(k-2) l+1$, it follows that $k \geqslant 4$. Then,

$$
\begin{aligned}
2((k-2) l+1)-(k-1)(l+1) & =2 k l-4 l+2-k l+l-k+1 \\
& =k l-3 l-k+3 \\
& =(k-3) l-(k-3) \\
& =(k-3)(l-1) \\
& >0 .
\end{aligned}
$$

Therefore $2((k-2) l+1)>(k-1)(l+1)$. Moreover, since $S_{k} \cap \mathbb{Z} / 3 \mathbb{Z}=\{a\}$, the $2((k-2) l+1)$ elements 0 and $2 a$ of $\pi_{3}(S)$ must be in $S_{1} \cup S_{2} \cup \cdots \cup S_{k-1}$. But we know that $\left|S_{i}\right| \leqslant l+1$, for all $1 \leqslant i \leqslant k-1$, in contradiction with $2((k-2) l+1)>(k-1)(l+1)$. We have proved that, in every cases, we have $\left|S_{i}\right| \leqslant l+1$, for all $1 \leqslant i \leqslant k$. We deduce that

$$
3((k-2) l+1)=\left|\pi_{3}(S)\right| \leqslant k(l+1) .
$$


Finally, since

$$
\begin{aligned}
3((k-2) l+1)-k(l+1) & =3 k l-6 l+3-k l-k \\
& =2 k l-6 l-k+3 \\
& =k(2 l-1)-3(2 l-1) \\
& =(k-3)(2 l-1),
\end{aligned}
$$

we obtain that $3((k-2) l+1)>k(l+1)$ for $k \geqslant 4$, in contradiction with $\left|\pi_{3}(S)\right| \leqslant k(l+1)$. For $k=3$, we have $\left|S_{1}\right|=\left|S_{2}\right|=\left|S_{3}\right|=l+1$. Since $\mathfrak{m}_{\pi_{3}(S)}(0)=l+1>l$, it follows from Lemma 5.1 that the number of multisets $S_{i}$ containing elements $0 \in \mathbb{Z} / 3 \mathbb{Z}$ is at least of two. It is exactly two because if each multiset $S_{i}$ contains elements $0 \in \mathbb{Z} / 3 \mathbb{Z}$, then there is a weakly $l$-sum-free multiset $S_{i}$ which contains all the elements of $\mathbb{Z} / 3 \mathbb{Z}$ and with $\left|S_{i}\right|=l+1$, in contradiction with Lemma 5.1. So we deduce that the partition $P$ is either of the form

$$
S_{1} \cap \mathbb{Z} / 3 \mathbb{Z}=S_{2} \cap \mathbb{Z} / 3 \mathbb{Z}=\{0, a\} \quad \text { and } \quad S_{3} \cap \mathbb{Z} / 3 \mathbb{Z}=\{2 a\},
$$

or of the form

$$
S_{1} \cap \mathbb{Z} / 3 \mathbb{Z}=\{0, a\}, \quad S_{2} \cap \mathbb{Z} / 3 \mathbb{Z}=\{0,2 a\} \quad \text { and } \quad S_{3} \cap \mathbb{Z} / 3 \mathbb{Z}=\{a, 2 a\},
$$

where $a \in\{1,2\} \subset \mathbb{Z} / 3 \mathbb{Z}$ and $\left|S_{1}\right|=\left|S_{2}\right|=\left|S_{3}\right|=l+1$. For the first form, we know from Lemma 5.2 that $S_{1}$ and $S_{2}$ are weakly $l$-sum-free if and only if $\sum_{x \in S_{1}} x=\sum_{x \in S_{2}} x=a$, that is, if and only if $\mathfrak{m}_{S_{1}}(a) \equiv \mathfrak{m}_{S_{2}}(a) \equiv 1 \bmod 3$. It follows that

$$
l+1=\mathfrak{m}_{\pi_{3}(S)}(a)=\mathfrak{m}_{S_{1}}(a)+\mathfrak{m}_{S_{2}}(a) \equiv 2 \quad(\bmod 3),
$$

and thus $l \equiv 1 \bmod 3$, in contradiction with the hypothesis that $l \equiv 0$ or $2 \bmod 3$. For the second form, we know from Lemma 5.2 that $S_{1}$ and $S_{2}$ are weakly $l$-sum-free if and only if $\sum_{x \in S_{1}} x=a$ and $\sum_{x \in S_{2}} x=2 a$, that is, if and only if $\mathfrak{m}_{S_{1}}(a) \equiv \mathfrak{m}_{S_{2}}(a) \equiv 1 \bmod 3$. Moreover, we have $\mathfrak{m}_{S_{2}}(2 a)=l+1-\mathfrak{m}_{S_{3}}(2 a)=\mathfrak{m}_{S_{3}}(a)$. It follows that

$$
l+1=\mathfrak{m}_{\pi_{3}(S)}(a)=\mathfrak{m}_{S_{1}}(a)+\mathfrak{m}_{S_{3}}(a)=\mathfrak{m}_{S_{1}}(a)+\mathfrak{m}_{S_{2}}(2 a) \equiv 2 \quad(\bmod 3),
$$

and thus $l \equiv 1 \bmod 3$, in contradiction with the hypothesis that $l \equiv 0$ or $2 \bmod 3$. This concludes the proof.

Claim 5.13. $\mathrm{WS}_{3}(k, l) \geqslant 3(k-2) l+2$ for $k \geqslant 3$ and $l \equiv 0,2 \bmod 3$.

Proof. Let $S=\{1,2, \ldots, 3(k-2) l+2\}$. We consider the following partition $P=$ $\left\{S_{1}, \ldots, S_{k}\right\}$ of $\pi_{3}(S)$ and we prove that it is weakly $l$-sum-free.

\begin{tabular}{|c|c|c|c|c|}
\hline$M$ & $\mathfrak{m}_{M}(0)$ & $\mathfrak{m}_{M}(1)$ & $\mathfrak{m}_{M}(2)$ & $|M|$ \\
\hline \hline$S_{1}$ & 0 & $(k-2) l+1$ & 0 & $(k-2) l+1$ \\
\hline$S_{2}$ & 0 & 0 & $(k-2) l+1$ & $(k-2) l+1$ \\
\hline$S_{3}, \ldots, S_{k}$ & $l$ & 0 & 0 & $l$ \\
\hline \hline$\pi_{3}(S)$ & $(k-2) l$ & $(k-2) l+1$ & $(k-2) l+1$ & $3(k-2) l+2$ \\
\hline
\end{tabular}


First, $P$ is a partition of $\pi_{3}(S)$ since the multiplicity functions verify that $\sum_{i=1}^{k} \mathfrak{m}_{S_{i}}(x)=$ $\mathfrak{m}_{\pi_{3}(S)}(x)$ for all $x \in \mathbb{Z} / 3 \mathbb{Z}$. The multisets $S_{1}$ and $S_{2}$ are weakly $l$-sum-free because, as already remarked above, a multiset which is only constituted by elements 1 or $2 \in \mathbb{Z} / 3 \mathbb{Z}$ is always weakly $l$-sum-free when $l \equiv 0,2 \bmod 3$. For $S_{3}, \ldots, S_{k}$, multisets containing only $l$ elements are always weakly $l$-sum-free in $\mathbb{Z} / 3 \mathbb{Z}$. This completes the proof.

\subsection{For $k \geqslant 3$ and $l \equiv 1 \bmod 3$}

Let $k \geqslant 3$ and $l \geqslant 2$ be two positive integers, with $l \equiv 1 \bmod 3$. We will prove that

$$
\mathrm{WS}_{3}(k, l)= \begin{cases}k(l+1) & \text { for } \quad\left\{\begin{array}{l}
k=3, k \geqslant 5 \text { and } l \geqslant 2, l \equiv 1 \bmod 3 \\
k=4 \text { and } l \geqslant 2, l \equiv 1,7 \bmod 9
\end{array}\right. \\
4 l+3 & \text { for } \quad k=4 \text { and } l \equiv 4 \bmod 9 .\end{cases}
$$

Claim 5.14. $\mathrm{WS}_{3}(k, l) \leqslant k(l+1)$ for $k \geqslant 1$ and $l \geqslant 2, l \equiv 1 \bmod 3$.

Proof. Directly follows from Lemma 5.1.

Claim 5.15. Let $l \equiv 1 \bmod 3$. Let $M$ be a multiset of $\mathbb{Z} / 3 \mathbb{Z}$ such that $|M \cap \mathbb{Z} / 3 \mathbb{Z}|=2$ and $|M|=l+1$. Then, $M$ is weakly l-sum-free if and only if $\mathfrak{m}_{M}(x) \equiv 1 \bmod 3$ for all $x \in M \cap \mathbb{Z} / 3 \mathbb{Z}$.

Proof. Suppose that $M \cap \mathbb{Z} / 3 \mathbb{Z}=\{a, b\}$ with $a \neq b$. First, since $l \equiv 1 \bmod 3$, it follows that

$$
\mathfrak{m}_{M}(a)+\mathfrak{m}_{M}(b)=l+1 \equiv 2 \quad(\bmod 3) .
$$

Thus either $\mathfrak{m}_{M}(a) \equiv \mathfrak{m}_{M}(b) \equiv 1 \bmod 3$ or $\mathfrak{m}_{M}(a) \equiv 0 \bmod 3$ and $\mathfrak{m}_{M}(b) \equiv 2 \bmod 3$. Suppose that $\mathfrak{m}_{M}(a) \equiv 0 \bmod 3$ and $\mathfrak{m}_{M}(b) \equiv 2 \bmod 3$. We know, by Lemma 5.2 , that

$$
\sum_{x \in M} x=\mathfrak{m}_{M}(a) \cdot a+\mathfrak{m}_{M}(b) \cdot b=2.2(a+b)=a+b .
$$

This leads to the equality

$$
2 b=0 . a+2 . b=\mathfrak{m}_{M}(a) \cdot a+\mathfrak{m}_{M}(b) \cdot b=a+b,
$$

in contradiction with $a \neq b$. This completes the proof.

Claim 5.16. $\mathrm{WS}_{3}(k, l) \geqslant \mathrm{WS}_{3}(k-3, l)+3(l+1)$ for $k \geqslant 4$ and $l \equiv 1 \bmod 3$.

Proof. Let $S=\left\{1,2, \ldots, \mathrm{WS}_{3}(k-3, l)\right\}$. Let $P=\left\{S_{1}, \ldots, S_{k-3}\right\}$ be a partition of $\pi_{3}(S)$ into weakly $l$-sum-free multisets of $\mathbb{Z} / 3 \mathbb{Z}$. Consider the multisets $S_{k-2}, S_{k-1}$ and $S_{k}$ of $\mathbb{Z} / 3 \mathbb{Z}$ defined below by their multiplicity function.

\begin{tabular}{|c|c|c|c|c|}
\hline$M$ & $\mathfrak{m}_{M}(0)$ & $\mathfrak{m}_{M}(1)$ & $\mathfrak{m}_{M}(2)$ & $|M|$ \\
\hline \hline$S_{k-2}$ & $l$ & 1 & 0 & $l+1$ \\
\hline$S_{k-1}$ & 0 & $l$ & 1 & $l+1$ \\
\hline$S_{k}$ & 1 & 0 & $l$ & $l+1$ \\
\hline \hline$S_{k-2} \cup S_{k-1} \cup S_{k}$ & $l+1$ & $l+1$ & $l+1$ & $3(l+1)$ \\
\hline
\end{tabular}


Then $P^{\prime}=\left\{S_{1}, \ldots, S_{k}\right\}$ is a partition of the multiset $\pi_{3}\left(S^{\prime}\right)=\pi_{3}\left(\left\{1,2, \ldots, \mathrm{WS}_{3}(k-3, l)+\right.\right.$ $3(l+1)\})$ since $\sum_{i=1}^{k} \mathfrak{m}_{S_{i}}(x)=\mathfrak{m}_{\pi_{3}(S)}(x)+(l+1)=\mathfrak{m}_{\pi_{3}\left(S^{\prime}\right)}(x)$ for all $x \in \mathbb{Z} / 3 \mathbb{Z}$. Moreover, since $l \equiv 1 \bmod 3$, it follows that the multisets $S_{k-2}, S_{k-1}$ and $S_{k}$ are weakly $l$-sum-free in $\mathbb{Z} / 3 \mathbb{Z}$ by Lemma 5.2 and Claim 5.15. Therefore $\mathrm{WS}_{3}(k, l) \geqslant \mathrm{WS}_{3}(k-3, l)+3(l+1)$ for all $k \geqslant 4$ and $l \equiv 1 \bmod 3$.

Claim 5.17. $\mathrm{WS}_{3}(3, l) \geqslant 3(l+1)$ for $l \equiv 1 \bmod 3$.

Proof. Let $S=\{1,2, \ldots, 3(l+1)\}$. The following 3-partition $P=\left\{S_{1}, S_{2}, S_{3}\right\}$ of $\pi_{3}(S)$ is weakly $l$-sum-free, as already seen in the proof of Claim 5.16.

\begin{tabular}{|c|c|c|c|c|}
\hline$M$ & $\mathfrak{m}_{M}(0)$ & $\mathfrak{m}_{M}(1)$ & $\mathfrak{m}_{M}(2)$ & $|M|$ \\
\hline \hline$S_{1}$ & $l$ & 1 & 0 & $l+1$ \\
\hline$S_{2}$ & 0 & $l$ & 1 & $l+1$ \\
\hline$S_{3}$ & 1 & 0 & $l$ & $l+1$ \\
\hline \hline$\pi_{3}(S)$ & $l+1$ & $l+1$ & $l+1$ & $3(l+1)$ \\
\hline
\end{tabular}

This completes the proof.

Let $k$ be a positive integer and let $S=\{1,2, \ldots, k(l+1)\}$. In the sequel of this section, suppose that there exists a partition $P=\left\{S_{1}, \ldots, S_{k}\right\}$ of $\pi_{3}(S)$ into $k$ weakly $l$-sum-free multisets of $\mathbb{Z} / 3 \mathbb{Z}$. By Lemma 5.1, $\left|S_{i}\right|=l+1$ and $\left|S_{i} \cap \mathbb{Z} / 3 \mathbb{Z}\right|=2$ for all $1 \leqslant i \leqslant k$. For every $x \in \mathbb{Z} / 3 \mathbb{Z}$, denote by $n_{x}$ the number of multisets $S_{i}$ in $P$ such that $x \in S_{i} \cap \mathbb{Z} / 3 \mathbb{Z}$. For $S_{i} \cap \mathbb{Z} / 3 \mathbb{Z}=\{a, b\}$, the multiset $S_{i}$ is weakly $l$-sum-free if and only if

$$
\mathfrak{m}_{S_{i}}(a) \equiv \mathfrak{m}_{S_{i}}(b) \equiv 1 \quad(\bmod 3)
$$

by Lemma 5.2 and Claim 5.15. It follows that

$$
n_{x} \in\{1, \ldots, k\} \quad \text { and } \quad n_{x} \equiv \mathfrak{m}_{\pi_{3}(S)}(x) \quad(\bmod 3),
$$

for all $x \in \mathbb{Z} / 3 \mathbb{Z}$. We distinguish different cases depending on the value of $k$ and the residue class of $l$ modulo 9 .

Claim 5.18. $\mathrm{WS}_{3}(5, l) \geqslant 5(l+1)$ for $l \equiv 4 \bmod 9$.

Proof. Consider the euclidean division of $l$ by 9 , that is $l=9 r+4$. Then,

$$
5(l+1)=5(9 r+5)=45 r+25=3(15 r+8)+1
$$

and

$$
\begin{array}{ll}
n_{0} \equiv \mathfrak{m}_{\pi_{3}(S)}(0)=15 r+8 \equiv 2 & (\bmod 3), \\
n_{1} \equiv \mathfrak{m}_{\pi_{3}(S)}(1)=15 r+9 \equiv 0 & (\bmod 3), \\
n_{2} \equiv \mathfrak{m}_{\pi_{3}(S)}(2)=15 r+8 \equiv 2 & (\bmod 3) .
\end{array}
$$

We deduce that $n_{0}, n_{2} \in\{2,5\}$ and $n_{1}=3$. Moreover, we have $n_{0}+n_{1}+n_{2}=10$. For $n_{0}=5, n_{1}=3$ and $n_{2}=2$, we can verify by using Lemma 5.2 and Claim 5.15 that the following partition of $\pi_{3}(S)$ is weakly $l$-sum-free. 


\begin{tabular}{|c||c|c|c||c|}
\hline$M$ & $\mathfrak{m}_{M}(0)$ & $\mathfrak{m}_{M}(1)$ & $\mathfrak{m}_{M}(2)$ & $|M|$ \\
\hline \hline$S_{1}$ & $9 r+4$ & 1 & 0 & $l+1$ \\
\hline$S_{2}$ & $3 r+1$ & $6 r+4$ & 0 & $l+1$ \\
\hline$S_{3}$ & 1 & $9 r+4$ & 0 & $l+1$ \\
\hline$S_{4}$ & $3 r+1$ & 0 & $6 r+4$ & $l+1$ \\
\hline$S_{5}$ & 1 & 0 & $9 r+4$ & $l+1$ \\
\hline \hline$\pi_{3}(S)$ & $15 r+8$ & $15 r+9$ & $15 r+8$ & $5(l+1)$ \\
\hline
\end{tabular}

This completes the proof.

Claim 5.19. $\mathrm{WS}_{3}(5, l) \geqslant 5(l+1)$ for $l \equiv 7 \bmod 9$.

Proof. Consider the euclidean division of $l$ by 9 , that is $l=9 r+7$. Then,

$$
5(l+1)=5(9 r+8)=45 r+40=3(15 r+13)+1
$$

and

$$
\begin{array}{ll}
n_{0} \equiv \mathfrak{m}_{\pi_{3}(S)}(0)=15 r+13 \equiv 1 & (\bmod 3), \\
n_{1} \equiv \mathfrak{m}_{\pi_{3}(S)}(1)=15 r+14 \equiv 2 & (\bmod 3), \\
n_{2} \equiv \mathfrak{m}_{\pi_{3}(S)}(2)=15 r+13 \equiv 1 & (\bmod 3)
\end{array}
$$

We deduce that $n_{0}, n_{2} \in\{1,4\}$ and $n_{1} \in\{2,5\}$. Moreover, we have $n_{0}+n_{1}+n_{2}=10$. For $n_{0}=n_{2}=4$ and $n_{1}=2$, we can verify by using Lemma 5.2 and Claim 5.15 that the following partition of $\pi_{3}(S)$ is weakly $l$-sum-free.

\begin{tabular}{|c||c|c|c||c|}
\hline$M$ & $\mathfrak{m}_{M}(0)$ & $\mathfrak{m}_{M}(1)$ & $\mathfrak{m}_{M}(2)$ & $|M|$ \\
\hline \hline$S_{1}$ & $3 r+1$ & $6 r+7$ & 0 & $l+1$ \\
\hline$S_{2}$ & 0 & $9 r+7$ & 1 & $l+1$ \\
\hline$S_{3}$ & $9 r+7$ & 0 & 1 & $l+1$ \\
\hline$S_{4}$ & $3 r+4$ & 0 & $6 r+4$ & $l+1$ \\
\hline$S_{5}$ & 1 & 0 & $9 r+7$ & $l+1$ \\
\hline \hline$\pi_{3}(S)$ & $15 r+13$ & $15 r+14$ & $15 r+13$ & $5(l+1)$ \\
\hline
\end{tabular}

This completes the proof.

Claim 5.20. $\mathrm{WS}_{3}(4, l) \geqslant 4(l+1)$ for $l \equiv 1 \bmod 9$.

Proof. Consider the euclidean division of $l$ by 9 , that is $l=9 r+1$. Then,

$$
4(l+1)=4(9 r+2)=36 r+8=3(12 r+2)+2
$$

and

$$
\begin{array}{ll}
n_{0} \equiv \mathfrak{m}_{\pi_{3}(S)}(0)=12 r+2 \equiv 2 & (\bmod 3) \\
n_{1} \equiv \mathfrak{m}_{\pi_{3}(S)}(1)=12 r+3 \equiv 0 & (\bmod 3), \\
n_{2} \equiv \mathfrak{m}_{\pi_{3}(S)}(2)=12 r+3 \equiv 0 & (\bmod 3)
\end{array}
$$


We deduce that $n_{0}=2$ and $n_{1}=n_{2}=3$. Moreover, we have $n_{0}+n_{1}+n_{2}=8$. We can verify by using Lemma 5.2 and Claim 5.15 that the following partition of $\pi_{3}(S)$ is weakly l-sum-free.

\begin{tabular}{|c||c|c|c||c|}
\hline$M$ & $\mathfrak{m}_{M}(0)$ & $\mathfrak{m}_{M}(1)$ & $\mathfrak{m}_{M}(2)$ & $|M|$ \\
\hline \hline$S_{1}$ & $6 r+1$ & $3 r+1$ & 0 & $l+1$ \\
\hline$S_{2}$ & $6 r+1$ & 0 & $3 r+1$ & $l+1$ \\
\hline$S_{3}$ & 0 & $6 r+1$ & $3 r+1$ & $l+1$ \\
\hline$S_{4}$ & 0 & $3 r+1$ & $6 r+1$ & $l+1$ \\
\hline \hline$\pi_{3}(S)$ & $12 r+2$ & $12 r+3$ & $12 r+3$ & $4(l+1)$ \\
\hline
\end{tabular}

This completes the proof.

Claim 5.21. $\mathrm{WS}_{3}(4, l)=4 l+3$ for $l \equiv 4 \bmod 9$.

Proof. Consider the euclidean division of $l$ by 9 , that is $l=9 r+4$. Then,

$$
4(l+1)=4(9 r+5)=36 r+20=3(12 r+6)+2
$$

and

$$
\begin{array}{ll}
n_{0} \equiv \mathfrak{m}_{\pi_{3}(S)}(0)=12 r+6 \equiv 0 & (\bmod 3), \\
n_{1} \equiv \mathfrak{m}_{\pi_{3}(S)}(1)=12 r+7 \equiv 1 & (\bmod 3), \\
n_{2} \equiv \mathfrak{m}_{\pi_{3}(S)}(2)=12 r+7 \equiv 1 & (\bmod 3) .
\end{array}
$$

We deduce that $n_{0}=3$ and $n_{1}, n_{2} \in\{1,4\}$. Moreover, $n_{1}, n_{2} \neq 1$ since $\mathfrak{m}_{\pi_{3}(S)}(1)=$ $\mathfrak{m}_{\pi_{3}(S)}(2)=12 r+7>l+1$. Thus $n_{0}=3$ and $n_{1}=n_{2}=4$ in contradiction with $n_{0}+n_{1}+n_{2}=8$. This proves that

$$
\mathrm{WS}_{3}(4, l) \leqslant 4 l+3 \quad \text { for } l \equiv 4 \quad(\bmod 9) .
$$

Finally, from Claim 5.16 and Claim 5.4, we deduce that $\mathrm{WS}_{3}(4, l) \geqslant \mathrm{WS}_{3}(1, l)+3(l+1)=$ $4 l+3$. This completes the proof.

Claim 5.22. $\operatorname{WS}_{3}(7, l) \geqslant 7(l+1)$ for $l \equiv 4 \bmod 9$.

Proof. Consider the euclidean division of $l$ by 9 , that is $l=9 r+4$. Then,

$$
7(l+1)=7(9 r+5)=63 r+35=3(21 r+11)+2
$$

and

$$
\begin{array}{ll}
n_{0} \equiv \mathfrak{m}_{\pi_{3}(S)}(0)=21 r+11 \equiv 2 & (\bmod 3) \\
n_{1} \equiv \mathfrak{m}_{\pi_{3}(S)}(1)=21 r+12 \equiv 0 & (\bmod 3) \\
n_{2} \equiv \mathfrak{m}_{\pi_{3}(S)}(2)=21 r+12 \equiv 0 & (\bmod 3)
\end{array}
$$

We deduce that $n_{0} \in\{2,5\}$ and $n_{1}, n_{2} \in\{3,6\}$. Moreover, we have $n_{0}+n_{1}+n_{2}=14$. Since $\mathfrak{m}_{\pi_{3}(S)}(0)=21 r+11>18 r+8=2 l$, it follows that $n_{0}=5$. For $n_{0}=5, n_{1}=6$ and $n_{2}=3$, we can verify by using Lemma 5.2 and Claim 5.15 that the following partition of $\pi_{3}(S)$ is weakly $l$-sum-free. 


\begin{tabular}{|c||c|c|c||c|}
\hline$M$ & $\mathfrak{m}_{M}(0)$ & $\mathfrak{m}_{M}(1)$ & $\mathfrak{m}_{M}(2)$ & $|M|$ \\
\hline \hline$S_{1}$ & $9 r+4$ & 1 & 0 & $l+1$ \\
\hline$S_{2}$ & $9 r+4$ & 1 & 0 & $l+1$ \\
\hline$S_{3}$ & $3 r+1$ & $6 r+4$ & 0 & $l+1$ \\
\hline$S_{4}$ & 1 & $9 r+4$ & 0 & $l+1$ \\
\hline$S_{5}$ & 1 & 0 & $9 r+4$ & $l+1$ \\
\hline$S_{6}$ & 0 & $6 r+1$ & $3 r+4$ & $l+1$ \\
\hline$S_{7}$ & 0 & 1 & $9 r+4$ & $l+1$ \\
\hline \hline$\pi_{3}(S)$ & $21 r+11$ & $21 r+12$ & $21 r+12$ & $7(l+1)$ \\
\hline
\end{tabular}

This completes the proof.

Claim 5.23. $\mathrm{WS}_{3}(4, l) \geqslant 4(l+1)$ for $l \equiv 7 \bmod 9$.

Proof. Consider the euclidean division of $l$ by 9 , that is $l=9 r+7$. Then,

$$
4(l+1)=4(9 r+8)=36 r+32=3(12 r+10)+2
$$

and

$$
\begin{array}{ll}
n_{0} \equiv \mathfrak{m}_{\pi_{3}(S)}(0)=12 r+10 \equiv 1 & (\bmod 3), \\
n_{1} \equiv \mathfrak{m}_{\pi_{3}(S)}(1)=12 r+11 \equiv 2 & (\bmod 3), \\
n_{2} \equiv \mathfrak{m}_{\pi_{3}(S)}(2)=12 r+11 \equiv 2 & (\bmod 3)
\end{array}
$$

We deduce that $n_{0} \in\{1,4\}$ and $n_{1}=n_{2}=2$. Since $\mathfrak{m}_{\pi_{3}(S)}(0)=12 r+10>9 r+7=l$, it follows that $n_{0}=4$. For $n_{0}=4$ and $n_{1}=n_{2}=2$, we can verify by using Lemma 5.2 and Claim 5.15 that the following partition of $\pi_{3}(S)$ is weakly $l$-sum-free.

\begin{tabular}{|c||c|c|c||c|}
\hline$M$ & $\mathfrak{m}_{M}(0)$ & $\mathfrak{m}_{M}(1)$ & $\mathfrak{m}_{M}(2)$ & $|M|$ \\
\hline \hline$S_{1}$ & $3 r+4$ & $6 r+4$ & 0 & $l+1$ \\
\hline$S_{2}$ & $3 r+1$ & $6 r+7$ & 0 & $l+1$ \\
\hline$S_{3}$ & $3 r+4$ & 0 & $6 r+4$ & $l+1$ \\
\hline$S_{4}$ & $3 r+1$ & 0 & $6 r+7$ & $l+1$ \\
\hline \hline$\pi_{3}(S)$ & $12 r+10$ & $12 r+11$ & $12 r+11$ & $4(l+1)$ \\
\hline
\end{tabular}

This completes the proof.

Finally, we obtain that $\mathrm{WS}_{3}(k, l)=k(l+1)$ for all $k \geqslant 3$ and $l \geqslant 2$, with $l \equiv 1 \bmod 3$, except for $k=4$ and $l \equiv 4 \bmod 9$, by combining Claim 5.14, Claim 5.16, Claim 5.17, Claim 5.18, Claim 5.19, Claim 5.20, Claim 5.22 and Claim 5.23.

This concludes the proof of Theorem 1.4. 


\section{Conclusion and future work}

In this paper, the modular generalized Schur numbers $\mathrm{S}_{m}(k, l)$ and the modular generalized weak Schur numbers $\mathrm{WS}_{m}(k, l)$ have been explicitly determined for all positive integers $k$ and $l$ and for small moduli $m \in\{1,2,3\}$. Although the determination of the exact values of $\mathrm{S}_{m}(k, l)$ and of $\mathrm{WS}_{m}(k, l)$ seems to be much more difficult for moduli $m \geqslant 4$, we can hope to find nontrivial lower and upper bounds of these numbers.

\section{Acknowledgements}

We would like to thank Shalom Eliahou for his constructive comments and suggestions throughout the preparation of this paper.

\section{References}

[1] H.L. Abbott and D. Hanson. A problem of Schur and its generalizations. Acta Arith., 20:175-187, 1972.

[2] H.L. Abbott and E.T.H. Wang. Sum-free sets of integers. Proc. Amer. Math. Soc., 67:11-16, 1977.

[3] A. Beutelspacher and W. Brestovansky. Generalized Schur numbers. Lecture Notes in Math., 969:30-38, 1982.

[4] P.F. Blanchard, F. Harary and R. Reis. Partitions into sum-free sets. Integers, 6:Article A7, 2006.

[5] S. Eliahou, J.M. Marín, M.P. Revuelta and M.I. Sanz. Weak Schur numbers and the search for G.W. Walker's lost partitions. Comput. Math. Appl., 63:175-182, 2012.

[6] G. Exoo. A lower bound for Schur numbers and multicolor Ramsey numbers of $K_{3}$. Electron. J. Combin., 1:\#R8, 1994.

[7] H. Fredricksen and M.M. Sweet. Symmetric sum-free partitions and lower bounds for Schur numbers. Electron. J. Combin., 7:\#R32, 2000.

[8] R.K. Guy. Unsolved Problems in Number Theory (3rd ed.), Problem Books in Mathematics, Springer-Verlag, New York, 2004.

[9] R.W. Irving. An extension of Schur's theorem on sum-free partitions. Acta Arith., 25:55-64, 1973.

[10] B.M. Landman and A. Robertson. Ramsey theory on the integers. Student Mathematical Library 24, Providence, RI: American Mathematical Society (AMS), 2004.

[11] R. Rado. Studien zur Kombinatorik. Math. Z., 36:424-470, 1933.

[12] M.I. Sanz. Números de Schur y de Rado. Departamento de Matemática Aplicada I, Universidad de Sevilla, 2010.

[13] I. Schur. Über die Kongruenz $x^{m}+y^{m} \equiv z^{m}(\bmod p)$. Jahresber. Deutsch. Math.Verein., 25:114-117, 1916.

[14] W. Sierpinski. Elementary theory of numbers (2nd ed.), North-Holland Mathematical Library 31, North-Holland Publishing, Amsterdam, 1988. 